\title{
Refereeing the Public Health
}

\author{
Hosea H. Harvey ${ }^{*}$
}

\section{ABSTRACT:}

Between January 2009 and October 2013, 49 states and the District of Columbia passed laws focusing on mitigating the consequences of traumatic brain injuries (TBIs) in organized youth sports. Using historical, contextual, and empirical methods, this Article describes the content, goals, and structure of youth sports TBI laws, while hypothesizing about their underlying legislative logic and long-term public health consequences. The Article's empirical evidence suggests two key findings: first, that a dominant interest group, the National Football League, helped to define the problem and its associated solutions for the vast majority of states, thus curving the legislative story arc in favor of its policy prescriptions; second, that existing youth sports TBI laws are focused on secondary, not primary, prevention, and may thus shift attention away from more comprehensive solutions. Finally, the Article explains why such state laws will likely fail to substantially resolve the larger untackled problem-significantly reducing the overall rate and number of TBIs in youth sports. After explaining why existing state youth sports TBI laws fail to accomplish this broader goal, the Article queries whether alternative policy or public health measures might offer more robust solutions.

* Assistant Professor of Law and Political Science, Temple University, James E. Beasley School of Law. Ph.D. (Stanford University), J.D. (Stanford Law School). The author would like to thank The Robert Wood Johnson Foundation and the Center for Health Law, Policy, and Practice at Temple University for research support. The author thanks Temple colleagues and anonymous reviewers for providing helpful comments on an earlier version of this draft and specially thanks Rebecca Cole for her invaluable feedback and attention to detail. The author thanks Shilpa Kadoo, Stephanie Kammer, and Nikos Kaplanov for valuable research assistance. This article benefits from data first reported in Hosea Harvey, Reducing Traumatic Brain Injuries in Youth Sports: Youth Sports Traumatic Brain Injury State Laws, January 2009-December 2012, 103 AM. J. PUB. HEALTH 1249 (July 2013). 


\section{TABLE OF CONTENTS}

INTRODUCTION 69

I. The EPIDEMIOLOGY OF TBIS AND YOUTH CONCUSSIONS

A. TBI DiAGNOSIS AND RECOGNITION: AGREEMENT AND DIVERGENCE .. 72

B. A VAST BUT UNCERTAIN SCOPE 73

C. CONCUSSION ASSESSMENT 75

D. TReatment: Consensus AND Divergence about ShORT-Term CONSEQUENCES 76

E. IDENTIFICATION AND TREATMENT OF MULTIPLE TBIS .......................... 78

F. Ambiguous Evidence Leads to Ambiguous Policy LogiC............. 79

II. SCIENTIFIC EVIDENCE AND YOUTH SPORTS TBI LAWMAKING 81

III. RUSHING THE FIELD: YOUTH SPORTS TBI LAWMAKING (2009-2012).... 85

A. THE "GALVANIZING INCIDENT" MODEL OF POLICYMAKING 85

B. Evaluating Youth SpORTs TBI Laws-Data AND Methodology 88

C. A UNIFORM POLICY SOLUTION? 88

D. THE ROLE OF POLICY EXPERIMENTATION (PuRPOSEFUl AND ACCIDENTAL)

E. Substantive Policy InNovation: Evidence, Theory, and Practice 96

F. EVIDENCE, INFLUENCE, AND RUSHING: COMMON CAUSAL MECHANISMS IN YOUTH SPORTS TBI LAW POLICYMAKING

IV. Failures OF Existing Policy AND Potential Policy IMPROVEMENTS

A. MANDATORY AGgREGATE REPORTING AND FEEDBACK REGIMES ..... 105

B. MANDATORY INDIVIDUAL TRACKING METRICS .................................. 106

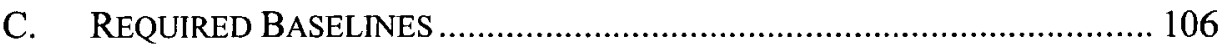

D. DiReCt INTERVENTION IN PARTICULAR SPORTS ................................ 107

E. FIXING THE REPORTING DISINCENTIVE........................................... 110

F. LEARNING FROM PROFESSIONAL SPORTS AND INDEPENDENT 


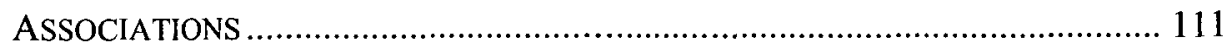

G. Evaluating Policy Alternatives .................................... 112

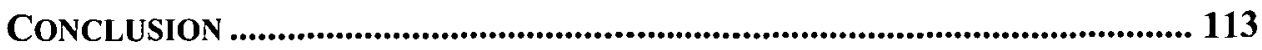

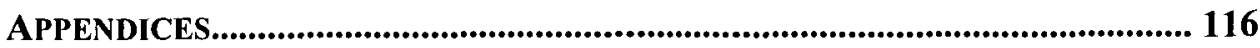

APPENDIX 1: EVIDENCE OF INFLUENCE-NEWS ................................ 116

APPENDIX 2: EVIDENCE OF INFLUENCE-LEGISLATIVE HISTORY ............. 117

APPENDIX 3: NFL STATEMENTS REGARDING ITS OWN LEGISLATIVE

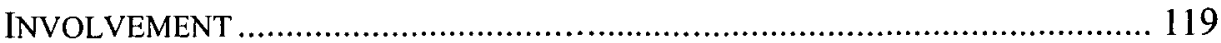




\section{INTRODUCTION}

In September 2010, Boston University's Center for the Study of Traumatic Encephalopathy ("the Center") found mild states of degenerative brain disease caused by repetitive concussions and/or sub-concussive brain injuries in a former football player. That the Center found such brain-trauma indicators after an athlete's death was not uncommon; its researchers have been at the forefront of studying professional football players for years. ${ }^{2}$ What was highly uncommon in this instance was that the Center found the indicators in a recently deceased college football player who had tragically committed suicide while still in college. $^{3}$ This discovery marked the first time that a college player's brain tissue had been systematically tested for the effects of repeated concussions, and it led to renewed efforts to bring concussion education and other reform efforts to youth sports.

In conjunction with another youth football player-related tragedy a year earlier, ${ }^{4}$ efforts to understand the short- and long-term effects of concussions or traumatic brain injuries (TBIs) broadened to include awareness of the effects of multiple traumatic brain injuries (MTBIs) on youth athletes. Because the scope of public awareness was magnified by the tragic experiences of these two young players, as well as publicized injuries in professional sports, a coalition of parents, educators, athletes, and professional groups used these events to marshal various constituencies, including legislatures, to address the problem. ${ }^{5}$ Despite the large number of interest groups galvanized by these events, a single interest group, the National Football League (NFL), soon emerged as the market leader for policy prescriptions addressing youth sports TBIs. With a myriad of statutory

2 The Center is considered the leading research organization focused on developing a comprehensive understanding of the long-term effects of concussions on athletes. Dozens of current and former NFL players are on its brain donation registry, and the NFL promotes this collaboration. See, e.g., Our Research, CTR. FOR The Study OF Traumatic Encephalopathy, Bos. UNIV., http://www-test.bu.edu/cste/our-research/ (last visited Dec. 2, 2013).

3 See Alan Schwarz, Suicide Reveals Signs of a Disease Seen in N.F.L., N.Y. TimEs, Sept. 13, 2010, http://www.nytimes.com/2010/09/14/sports/14football.html.

4 The case involves a Washington state middle-school football player named Zackery Lystedt, discussed below in Part III.A.

5 See, e.g., Ben McGrath, Does Football Have a Future?, New Yorker, Jan. 31, 2011. Broadly speaking, the high rate of youth concussions and public discussions thereof often focuses on youth football, which does account for a high portion of such injuries. In contrast, except where noted, this Article considers all youth sports where athletes are at risk for TBIs or MTBIs in its examination of the epidemiology and TBI-focused legal regimes. This scope includes all organized youth sports, particularly those with risks of head impact or injury. See generally Andrew Gardner et al., Chronic Traumatic Encephalopathy in Sport: A Systematic Review, BrIT. J. SpORTS MEd. (forthcoming) (manuscript at 1-6) (2013), available at http://bjsm.bmj.com/content/early/ 2013/06/25/bjsports-2013-092646.full.pdf (describing some new understandings of how sports TBIs occur outside of direct football head hits). 
and regulatory options available, state laws emerged as the quickest, if not the most effective, solution.

Public health law scholars have engaged in similar policymaking debates, rightly focused on the use of evidence and data when proposing new interventions, particularly those harnessing the force of law. These evidencebased interventions are not unique to public health, and have been widely utilized in other areas. ${ }^{6}$ Further, scholars agree that the ideal environment for implementing laws of the type discussed here would rely on accumulation of existing research, synthesis of that research into a set of discrete outcomes, translation of those outcomes into a policy framework, and an evaluation of that framework's ability to influence and modify the behavior or outcome it seeks to change. ${ }^{7}$ But with respect to youth sports TBI laws, this approach was not consistently followed. Nonetheless, lawmakers acted at a fairly quick pace.

Between January 2009 and October 2013, 49 states and the District of Columbia passed laws designed to minimize the consequences of TBIs in youth sports. ${ }^{8}$ Despite this impressive and sudden legislative response to a longstanding problem, the core logic and evidence supporting such laws has remained a mystery. This uncertainty about the link between science and policy should have led lawmakers to question the efficacy and efficiency of their efforts during this period. What do these laws purport to do? What are the appropriate behavioral and health-science logic models underpinning these legislative mandates? Will these laws have their purported desired effect-a reduction in instances and effects of youth TBIs? And if the laws do eventually have such an effect, how will policymakers, public-health officials, and other advocates know?

This Article is an attempt to answer some of these core questions in the absence of conclusive scientific evidence. By October 2013, although every state but Mississippi had passed some form of youth sports TBI law, policymakers, health advocates, and outside interest groups still had little information as to the expected effectiveness of these laws on reducing the overall number of youth TBIs and reducing the long-term effects of youth TBIs. ${ }^{9}$ As this Article explains, despite the recent proliferation of state laws, there is no short-term evidence (or

6 See, e.g., Ross C. Brownson et al., Understanding Evidence-Based Public Health Policy, 99 AM. J. Pub. Health 1576, 1576-83 (2009).

7 See, e.g., Scott Burris et al., Making the Case for Laws that Improve Health: A Framework for Public Health Law Research, 88 MiLBANK Q. 169 (2010).

8 For a discussion of laws and data-gathering methods, see Sections III.A and III.B below.

9 Scientific uncertainty notwithstanding, the sudden and swift implementation of the laws and their corresponding lack of evaluative metrics means that no systematic studies were undertaken to test their effectiveness prior to implementation. Therefore, this Article evaluates their theoretical goals, attempts to determine whether those goals can be met, and explores what might be done to further align the goals with existing scientific evidence. The Article focuses primarily on middleand high-school sports, since almost all existing state laws focus on that domain to the exclusion of others (recreational sports and college sports in particular). 
even a metric or framework) to evaluate whether such laws will accomplish their stated goals. Further, because many of these laws follow a familiar blueprint endorsed by the NFL and leading brain injury advocacy groups, there is little substantive policy experimentation between states and so it will be difficult to causally assess differences in state-level outcomes over time. This Article argues that since lawmakers were starting from scratch, they could have aimed for a more rigorous, engaged, and long-term TBI reduction solution, despite some scientific uncertainty about the epidemiology of youth TBIs.

This Article is also a study of a hyperkinetic form of state-level policymaking, and all available evidence suggests that the pace and consistency of such lawmaking can primarily be attributed to the role of outside interest groups, most notably the NFL. Evidence gathered here points toward the NFL's significant role in the promotion of a select group of public health law and policy prescriptions with respect to youth sports TBIs. As a result, it is hard to determine ex ante whether laws produced in such an influence-driven environment were designed to actually mitigate youth TBIs or to further the goals of various interest groups, which may not view TBI reduction as their primary objective. Ideally, each state could serve as a laboratory where one centralized, authorized, and motivated state-level regulator would take charge of developing a broad-based public health law and policy solution to combating TBIs in youth populations. ${ }^{10}$ No state has created such a centralized regime, and the role of interest groups - the NFL in particular-may have influenced that policy choice. The NFL's intervention may have led states to act, which is likely better than silence. At the same time, the NFL's role may also have caused states to accept an initial slate of recommendations as a final policy prescription, while ignoring nuanced issues of TBI causation and policy alternatives to the NFL's approach. It is not merely that states legislated in a scientific vacuum, but that alternative methodologies or interventions were not considered against the backdrop of the Lystedt Law and the NFL's support.

This Article proceeds as follows. First, the epidemiology of TBIs is analyzed with particular focus on youth populations-referencing adult and college populations when appropriate. " Next, the Article turns to public health law

$10 \mathrm{It}$ is common in public health lawmaking frameworks to analyze the effects of state laws in certain public health areas (tobacco, seat belts, alcohol, etc.) and then to frame the divergence of such laws as an opportunity to test the effectiveness of public health law innovations in the fifty state lawmaking laboratories. For the purposes of this Article, however, the utility of this framework is minimal because of the uniformity of laws across states.

11 The Article does not specifically focus on the robust literature involving collegiate or professional anti-concussion initiatives except as limited comparative frameworks for understanding the general epidemiology of TBIs. Instead, the Article's focus is on the nexus between the science of understanding youth TBIs, the legislative responses to that scientific understanding, and the connection between the epidemiology of youth TBIs and laws that could, if 
intervention efforts at the state level and analyzes youth sports TBI laws for key similarities and differences. The objective in this section is to highlight legal regimes that effectively incorporate the best scientific knowledge-and those that do not. After asking whether these laws are indeed effective in their purported key goal-reducing the incidence and consequences of TBIs in youth populations-the Article analyzes the role of the NFL in the policymaking process. Concluding that the NFL's role in shaping the legislative story arc was formidable, the Article then turns to analyzing the areas that state youth sports TBI laws chose to ignore, such as evaluative metrics and other direct outcome measures. After examining the failures of the state youth sports TBI laws, the Article turns to addressing a broader question: whether law is the proper forum to address this public health problem. Concluding that existing youth sports TBI laws are indeed necessary and acknowledging that these initial responses did more good than harm, the Article closes by focusing on ways in which new legislative initiatives can improve upon earlier interventions.

\section{THE EPIDEMIOLOGY OF TBIS AND YOUTH CONCUSSIONS}

\section{A. TBI Diagnosis and Recognition: Agreement and Divergence}

For purposes of this Article, a concussion or TBI is defined as (a) an injury to the head arising from blunt trauma, an acceleration force, or a deceleration force, (b) which then disrupts the normal functioning of the brain, (c) which causes the individual to exhibit one of many indicators (whether observed or self-reported), and (d) where any such indicator is attributable to the injury. ${ }^{12}$ More generally speaking, a concussion is a mild brain injury, caused by trauma to the head, which results in a temporary disruption of normal brain functions. ${ }^{13}$

Since a concussion typically involves some direct or indirect force to the head, observers usually recognize concussions in youth athletes following a perceived head impact or injury. Yet, there is no agreed-upon TBI diagnostic metric, and no uniform national TBI reporting protocols. ${ }^{14}$ As a result of this data

properly aligned, minimize their frequency and long-term impact.

12 Collectively, those concussion indicators include the trauma or force followed by (a) transient confusion, disorientation, or impaired consciousness; (b) dysfunction of memory; (c) loss of consciousness; or (d) signs of other neurological or neuropsychological dysfunction including seizures, irritability, lethargy, fuzzy vision, headache, or vomiting.

13 Even this simple definition is not uniformly accepted. Others suggest that body jolts and body blows that do not result in a literal "blow to the head" can cause concussions, because any major body blow can jostle the brain. While this is true, the focus here is on those concussions caused by direct head impacts.

14 Christopher Randolph et al., Concussion Symptom Inventory: An Empirically Derived Scale for Monitoring Resolution of Symptoms Following Sport-Related Concussion, 24 ARCHIVES Clinical Neuropsychology 219, 229 (2009). 
void, first-line responders to potential TBI events have settled on some commonly accepted-but scientifically imprecise-basic TBI detection methods. At the outset, the traditional method for first recognizing a potential TBI during a youth athletic practice or competition is through visual observation of an impact followed by visible manifestations of various TBI consequences or symptoms. Other players may notice the visual signs of a TBI, such as vomiting, loss of consciousness, or confusion-even when the symptoms are not understood directly by the youth athlete himself. Therefore, one key line of defense in recognizing TBIs is fellow athletes' awareness of typical symptoms.

One step removed, coaches and other team leaders' awareness is often a critical element in TBI diagnosis. To ensure that athletes receive the assistance they require following a TBI, the coach, athletic trainer, and/or other supervisory official should be trained to recognize the various signs and symptoms of a concussion. For example, if a coach does not know that an athlete exhibiting retrograde amnesia or confusion may have a TBI (and may be at risk for another), then the coach may not remove the player from the game, thereby risking further injury. Therefore, a coach's simple visual awareness and alertness on the field, coupled with a fundamental understanding of TBIs and their symptoms, can be critical in diagnosis.

Slightly more removed from initial direct observation, parents are another necessary line of defense. When parents are at competitions watching their child, they can serve as useful monitors to notice when their child or another young athlete is experiencing TBI symptoms. Parents can also be a counterweight in situations where the interests of coaches and players might not be fully aligned. If an injury goes unnoticed on the field, parents are best equipped to notice subtle changes in a young athlete's behavior after the competition and assess whether their son or daughter has experienced a TBI or requires medical attention. Therefore, parents' ability to serve as another safeguard against an injured player's further injury is predicated upon the parents' knowledge of concussive symptoms. Without this knowledge and understanding of TBI symptoms and the risk of further injury, many parents will not understand the symptoms their child may be experiencing; this may cause a potentially dangerous condition to go undiagnosed and untreated.

\section{B. A Vast but Uncertain Scope}

No one disagrees that youth are at risk of developing TBIs when they play organized sports. Instead, the discussion about youth and TBIs is characterized by scientific uncertainty about causality, the scope of youth concussion rates, effects of multiple impacts, treatment, evaluation, and assessment of long-term 
outcomes. ${ }^{15}$ It is difficult to be specific about each of these elements because there is no comprehensive reporting mechanism at the state or national level to identify all instances of youth TBIs during a given year or the percentage of those caused by youth sports. ${ }^{16}$ Therefore, it becomes necessary to rely on estimates, anecdotes, and/or incomplete reporting systems to establish baseline population data. When state laws attempt to minimize the number and consequences of TBIs in youth populations, all but a few do so without relying on a state-level evaluative metric or data-driven framework. It is thus difficult to imagine exactly how the success of these laws will be evaluated. Nonetheless, we do know that a problem of some magnitude exists, even if we are unsure of exactly how significant it is.

That said, experts generally believe that the prevalence of sports-related concussions among young people is significantly higher than reported. ${ }^{17}$ As identifying metrics and reporting mechanisms have improved, reports of concussions in youth sports have skyrocketed. But because reporting, definitions, treatment standards, and testing have also all improved during the past fifteen years, it is nearly impossible to be certain whether the data trends show an absolute rise or simply reflect more accurate assessment of incidences in youth populations. ${ }^{18}$ Most experts agree that children and teenagers are at a greater risk of concussion than adult populations and that concussions in younger people take longer to heal because their brains are still developing. Some estimates suggest that up to $10 \%$ of all high-school athletes in contact sports suffer a concussion during each season. ${ }^{19}$ Youth sports and bicycle accidents account for the majority of concussion cases among 5- to 14-year-olds. ${ }^{20}$ And somewhat recent estimates of youth sports concussions suggest that there are between 1.6 and 3 million (or

15 This Article does not purport to review the entire field of literature regarding youth and TBIs. For a brief sampling of work in the field, see Melissa L. McCarthy et al., Health-Related Quality of Life During the First Year After Traumatic Brain Injury, 160 ARCHIVES PEDIATRIC \& ADOLESCENT MED. 252, $260 \mathrm{nn} .12-26$ (2006).

16 Although reporting systems have improved over the last few years, the problem for our purposes is tracking longitudinal data about the effects of TBIs and MTBIs over time and across varied and stable populations. Because reporting mechanisms have historically been weak to nonexistent, such long-term analysis is not yet possible. See Bryan Jennett, Epidemiology of Head Injury, 60 J. Neurology, Neurosurgery \& Psychiatry 362, 364 (1996) (noting that "data on admissions for head injury are not routinely collected on a national basis in the United States").

17 Steven T. DeKosky et al., Traumatic Brain Injury-Football, Warfare, and Long-Term Effects, 363 NEW ENG. J. MED. 1293, 1295 (2010).

18 Mike Mitka, Reports of Concussions From Youth Sports Rise Along With Awareness of the Problem, 304 JAMA 1775, 1775-76 (2010).

19 See Concussion Facts, SPORTS CONCUSSION INST., http://www.concussiontreatment.com/ concussionfacts.html (last visited Dec. 2, 2013).

20 Allan H. Ropper \& Kenneth C. Gorson, Concussion, 356 New Eng. J. Med. 166 (2007). Bicycling, in addition to being regulated under other laws, is not considered a "youth sport" for the purposes of this Article and shall not be discussed in depth. 
more) annually in the United States. ${ }^{21}$

However, because specific data are lacking at both the national and state level, experts acknowledge a high degree of uncertainty notwithstanding their general agreement about the significance of the problem. For example, studies provide estimates ranging from a 200 to $300 \%$ increase in reported instances within the last decade. ${ }^{22}$ Some provide generalized estimates across all sports while others provide estimates specific to certain types of sports. ${ }^{23}$ Part of the uncertainty in these estimates is based on the fact that the only uniform national reporting regime, the National Electronic Injury Surveillance System All Injury Program (NEISS-AIP), relies on a highly limited number of reporting hospitals. But the statistics, whether they over-or underestimate, indicate that the problem exists in sufficient magnitude to warrant attention. ${ }^{24}$

\section{Concussion Assessment}

Even though most assessments of whether an individual has suffered a TBI are not robustly scientific, this does not mean that more scientific measurements and assessments are impossible. Best practices in the identification of TBIs involve pre-competition baseline measurements of an athlete's overall brain function. Companies, such as $\operatorname{ImPACT}{ }^{25}$ and CogState Sport, ${ }^{26}$ and healthcare institutions such as the Mayo Clinic ${ }^{27}$ offer computerized baseline testing. Once baseline information is recorded, the results can be interpreted by comparing them with an injured athlete's post-injury responses to determine if he or she demonstrates cognitive symptoms that would suggest a concussion. ${ }^{28}$ Generally, developers advertise these tests as low-cost assessment tools that can be administered by a healthcare professional ${ }^{29}$ or on-site at an athletic facility

21 Jean A. Langlois et al., The Epidemiology and Impact of Traumatic Brain Injury: A Brief Overview, 21 J. Head Trauma Rehab. 375, 375-78 (2006).

22 Ropper \& Gorson, supra note 20.

23 Luke M. Gessel et al., Concussions Among United States High School and Collegiate Athletes, 42 J. ATHLETIC TRAINING 495, 497 (2007) (providing both overall and sport-specific estimates).

24 There are many more studies spanning decades of research. This Article seeks to summarize broad research themes without purporting to be the definitive scientific review of all extant TBI literature.

25 See IMPACT, http://www.impacttest.com (last visited Dec. 2, 2013).

26 See CoGSTATE, http://www.cogstate.com (last visited Dec. 2, 2013).

27 See Susan Shepherd, Mayo Clinic Offers Baseline Concussion Testing to Arizona High School Athletes, MAYO CLINIC NEws, June 23, 2011, http://newsblog.mayoclinic.org/201 1/06/23/mayo-clinic-offers-baseline-concussion-testing-toarizona-high-school-athletes.

28 Id.

29 See Frequently Asked Questions, IMPACT, http://impacttest.com/about/?Frequently-AskedQuestions-7 (last visited Dec. 2, 2013). 
through the use of a computer or tablet device. ${ }^{30}$ These computerized tools provide coaches and staff with another diagnostic metric that can be used to prevent youth athletes with TBIs from prematurely returning to play and risking further injury. For example, the Mayo Clinic's test can be taken from any computer with Internet access, takes 8 to 15 minutes to complete, and allows the athlete or parent to share the results with their chosen healthcare providers. These tools are not meant to be used in isolation, and their results may require health provider intervention. The Mayo Clinic recommends use of its test in conjunction with a comprehensive medical evaluation when an athlete is suspected of having a concussion. ${ }^{31}$ In those cases where baseline tests reveal abnormalities or where other indicators point to TBI complications, the athlete would then be directed to receive medical treatment and preventive measures. Notwithstanding the provider of the test or its ease of use, for a variety of reasons-cost likely among them-few states require baseline testing for any of their student athletes.

\section{Treatment: Consensus and Divergence about Short-Term Consequences}

Once an athlete is suspected of having a TBI, removal from competition is obviously the first immediate form of treatment and prevention of further injury. However, while there is some initial consensus about immediate first steps, there is substantial disagreement about actions that should follow.

Generally speaking, initial treatment of a TBI involves assessing the severity of the injury and monitoring the athlete's condition. Depending on the severity of the injury, many athletes will require rest until their concussive symptoms have subsided. Recommendations found in the National Athletic Trainers' Association Position Statement: Management of Sport-Related Concussion provide that a coach, athletic trainer, or physician should monitor an athlete with a concussion at " 5 -minute intervals from the time of the injury until the athlete's condition completely clears or the athlete is referred for further care." ${ }^{32}$ When the concussion results in loss of consciousness or amnesia lasting longer than 15 minutes, the athlete will require medical treatment to prevent additional complications. ${ }^{33}$ Similarly, the onset of drowsiness, paralysis, or language impairment after a concussion is cause for concern about the possibility of more serious complications, usually warranting swift medical examination and medical imaging studies. ${ }^{34}$ Nonetheless, despite much of what we do know about TBIs,

30 See There's No Such Thing as a Tough Brain, KIng DEvick TEST, http://kingdevicktest.com/ for-concussions/ (last visited Dec. 2, 2013).

31 See Shepherd, supra note 27.

32 Kevin M. Guskiewicz et al., National Athletic Trainers' Association Position Statement: Management of Sport-Related Concussion, 39 J. ATHLETIC TRAINING 280, 282 (2004).

33 Id.

34 See Ropper \& Gorson, supra note 20, at 168. 
many rules about medical treatment (for example, requiring a CT scan for an injured athlete) are based on antiquated science and studies of an age-dissimilar population, which calls into question their generalizability to youth populations. ${ }^{35}$ Therefore, additional study is required to determine whether current treatment standards are appropriate for youth with TBI symptoms. ${ }^{36}$

Furthermore, because an initial TBI puts an athlete at greater immediate risk of suffering another, an important part of treatment is prevention of another immediate TBI-specifically, by ensuring that the athlete does not return to competition too quickly following the initial head injury. Although there is rarely unanimity in medical opinion with respect to TBIs, all recommendations for TBI treatment identify removal from potential TBI-inducing activity in the period following an initial TBI as the most critical action to prevent compounding the initial brain injury. During the period immediately following a concussion, a subsequent TBI can have disproportionately severe health consequences, no matter how mild the initial concussion. ${ }^{37}$ Therefore, as standards have evolved, removing athletes from competition for at least an entire day has become a de facto minimum standard to prevent further aggravation of the initial injury.

Finally, treatment of a TBI must fully account for the array of residual effects of TBIs, which generally include continuation of concussion-related symptoms long after the injury-known as post-concussion syndrome. Post-concussion syndrome is "a constellation of sometimes disabling symptoms, mainly headache, dizziness, and trouble concentrating, in the days and weeks following concussion." 38 These symptoms can last for as little as a few days to as long as one year or more. ${ }^{39}$ As with other epidemiological matters pertaining to concussions, there is a lack of data from controlled trials for guidance on treatment of post-concussion syndrome, and there is some indication that the extent and duration of some symptoms may be psychosomatic. ${ }^{40}$ Nonetheless, more research is required to more fully understand the scope of the problem.

35 See Micelle J. Haydel et al., Indications for Computed Tomography in Patients with Minor Head Injury, 343 NEw ENG. J. MED. 100, 101-02 (2000) (noting that the criteria requiring a CT scan include Glasgow Coma Scale of 15, headache, vomiting, age above 60 years, drug or alcohol intoxication, persistent anterograde amnesia, evidence of traumatic soft-tissue or bone injury above the clavicle, or seizure).

36 Youth were excluded from the original studies, and we now know that youth brains respond differently to concussive events and are still forming - unlike the populations then studied. See Ropper \& Gorson, supra note 20, at 168.

37 In rare cases, a second impact could cause permanent injury or death.

38 See Ropper \& Gorson, supra note 20, at 169.

$39 \mathrm{Id}$.

$40 \mathrm{Id}$. 


\section{E. Identification and Treatment of Multiple TBIs}

Many professional athletes who suffer MTBIs eventually experience severe health consequences, such as ALS and Chronic Traumatic Encephalopathy (CTE) ${ }^{41}$ American football remains the primary focus of CTE research because of post-mortem access to a player's brain tissue, which must be analyzed at the cellular level using specialized proteomics technology to definitively study MTBI consequences. ${ }^{42}$

MTBIs are considered one cause of CTE, which is a progressive degenerative disease of the brain that has been found in athletes (and others) with a history of repetitive brain trauma. ${ }^{43}$ Yet the uncertainty about the long-term impact of MTBIs also exists with respect to CTE; scientists are not exactly sure how CTE ultimately manifests. ${ }^{44}$ In addition to this uncertainty, there have not yet been any longitudinal cohort studies that evaluate long-term health outcomes by following athletes with and without TBIs over a multi-decade span. ${ }^{45}$ The absence of such studies makes it impossible to precisely determine the causal relationship, if any, between youth-sports injuries, professional sports injuries, and the subsequent early-onset dementia that has been observed in some former professional athletes. ${ }^{46}$

For many reasons, we simply do not know much about the specific long-term effects of repeated concussions suffered in youth sports. Perhaps one factor in

41 The evolution of science with respect to CTE is fairly remarkable. The discovery began in 2002 by identifying a complex new form of post-concussive dementia, recognized in an autopsy of former NFL player Mike Webster and defined as CTE. Since then, scientific agreement about the phenomenon has coalesced, as CTE was later identified in former Philadelphia Eagles player Andre Waters (who was posthumously diagnosed after committing suicide following numerous concussions), former Houston Oilers linebacker John Grimsley (who died from a self-inflicted gunshot wound in 2008), and many others. Some players, such as Sean Morey, have been diagnosed as having a high likelihood of developing CTE and have retired in an effort to prevent additional harm.

42 See Field Hearing: Legal Issues Relating to Football Head Injuries Before the H. Comm. on the Judiciary, Part II, 111 th Cong. (2010) (statement of Bennet I. Omalu, Clinical Professor of Psychology, University of California, Davis) [hereinafter Field Hearing]. It appears that the Center's public mission and the media visibility of its findings have caused other professional football players to consider brain donation.

43 See, e.g., What is CTE?, CTr. for the Study of Traumatic Encephalopathy, Bos. UNIV., http://www-test.bu.edu/cste/about/what-is-cte/ (last visited Dec. 5, 2013).

44 One explanation of CTE development appears as follows: mild trauma to brain causes axons to be sheared, diffuses leakages across membranes, tau formation (possibly in genetically predisposed people). See Field Hearing, supra note 42.

45 BU's CTE Center has proposed the first such comprehensive study, but its recruitment is still ongoing. See, e.g., Clinical Studies, CTr. For the Study of Traumatic Encephalopathy, Bos. UNIV., http://www-test.bu.edu/cste/our-research/clinical-studies/ (last visited Dec. 5, 2013).

46 Kevin M. Guskiwicz et al., Recurrent Concussion and Risk of Depression in Retired Professional Football Players, 39 MED. \& SCI. IN SPORTS \& EXERCISE 903 (2007). 
our lack of knowledge is the failure of key constituencies to agree on a system for youth athlete TBI reporting and tracking over time. ${ }^{47} \mathrm{~A}$ second key reason is that long-term effects can only be truly studied by analyzing brain tissue. ${ }^{48}$ Consequently, athletes cannot be examined for long-term effects during their lifetimes, and those who are examined after death tend to be athletes who played professional sports, particularly football. The athletic careers of professional sports players are obviously very different from those of most youth athletes. It is difficult to make inferences about the impact of MTBIs on young amateur athletes from evidence drawn from professionals. ${ }^{49}$ For these reasons, while there is agreement that MTBIs (especially within a short time) can result in short-and long-term health consequences for youth, there is still much to learn about the scope of the phenomenon and its consequences. ${ }^{50}$

\section{F. Ambiguous Evidence Leads to Ambiguous Policy Logic}

As the preceding sections have described, scientific evidence regarding virtually every aspect of the scope and consequences of TBIs is highly underdeveloped. This is especially true with respect to youth involved in school sports. There are no large-scale studies that have measured the impact of public health law interventions in this area. The new state-level legislation discussed in this Article constitutes a type of policymaking driven by news events and studies of unique, individual experiences. Such legislation by anecdote works when empirics are lacking, because their absence creates a vacuum in which any policy seems better than none. ${ }^{51}$ While legislating by anecdote has clearly obtained

47 This failure persists for reasons that confound most experts. In October 2013, the Institute of Medicine of the National Academies released the results of its national expert report on youth sports TBIs, concluding that failure to track incidence data was harmful to the goal of reducing TBIs and calling for the Centers for Disease Control and Prevention (CDC) to establish a national surveillance system. See, e.g., Sports-Related Concussions in Youth: Improving the Science, Changing the Culture, INST. OF MED. (Oct. 2013), http://www.iom.edu/ /media/Files/ Report\%20Files/2013/Concussions/concussions-RB.pdf [hereinafter Sports-Related Concussions].

48 There are certainly a variety of physiological methods and metrics by which health professionals can gather the information, but it appears that this more invasive technique objectively reveals more than the other methods.

49 As youth sports TBIs gain wider recognition as a public health issue, observational studies are being conducted by experts to begin collecting data regarding the specific effects of such repeated traumas on children. See, e.g., Second Concussion Means Longer Recovery Time, REDORBit.com (June 24, 2013), http://www.redorbit.com/news/video/health 2/1112874341/ recovery-from-second-concussion-061413/ (showing that youth athletes take longer to recover after their second or third concussion).

50 See Tareg Bey \& Brian Ostick, Second Impact Syndrome, W.J. EMERGENCY MED. 6, 6-10 (2009).

51 See Theresa Glennon, Choosing One: Resolving the Epidemic of Multiples in Assisted Reproduction, 55 VILL. L. REV. 147 (2010). 
rapid legislative results in the area of youth sports TBI laws, the value of laws that lack empirical underpinnings remains to be seen.

Despite the potentially problematic absence of empirical evidence, there are two broad areas of agreement about the consequences of youth concussions:

(1) At a minimum, doctors and others believe that youth are more at risk than adults for short- and long-term TBI effects because the brain is not fully formed until the late teen years moving into adulthood. ${ }^{52} \mathrm{As}$ such, experts conclude that injuries to a developing brain are likely to have more long-term consequences (and result in longer healing times) than injuries to a fully-formed brain; and

(2) Multiple concussions in a short time span likely lead to a more rapid onset of short- and long-term brain dysfunction.

Given these two broad areas of agreement, short-term cohort studies of athletes in the field (of which there are few) attempt to add clarity to the muddled causal links. ${ }^{53}$ These studies, which describe broad data that can lead to prescriptive measures, seek to provide generic universal recommendations. These generalized recommendations do not differentiate TBI reduction policy prescriptions and minimize the observed differences in TBI rates across diverse populations. ${ }^{54}$ By keeping a broad focus on injury prevention, such studies should point toward a broad public health intervention that encompasses preventing injury, recognizing injury, and caring for injury. However, most of these studies and the legal interventions derived from them focus on prevention of long-term damage from concussions rather than prevention of concussions themselves. Despite this failing, such studies at a minimum provide some broad empirical support for certain types of public health interventions that may positively affect public health with respect to youth populations. The next section discusses two of these broad studies before turning to state-level legislative efforts that have implemented or capitalized on some of the studies' general findings.

52 See Bey \& Ostick, supra note 50, at 6-10.

53 The studies are described in Part II below.

54 Although such studies show that incidences and rates can be separated and distinguished by key independent variables such as type of sport, gender, race, and income, policy prescriptions do not attempt to sort TBI reduction strategies by using such inter-group/sport differences to achieve more narrowly tailored TBI reduction goals. See, e.g., P. David Adelson \& Patrick M. Kochanek, Topical Review: Head Injury in Children, 13 J. CHILD NEuROLOGY 2 (1998) (providing a broad review of some demographic variance indicators). 


\section{SCIENTIFIC EVIDENCE AND YOUTH SPORTS TBI LAWMAKING}

Although no state's youth sports TBI law contains sex- or sport-based policy distinctions, youth concussions do vary substantially on both of these metrics. Few studies have attempted to sort out the causal factors behind these key differences and lay the groundwork for (a) explaining or reducing demographic variance in youth TBIs and (b) influencing how public health laws might reduce these injuries or mitigate their consequences. Instead, policy prescriptions rely on only the broadest of demographic details. ${ }^{55}$

Some recent studies have contributed much to our understanding of how demographic variables might change the incidence, rate, or outcomes of concussions. One such study (the "High School Trends Study") tracked twelve boys' and girls' sports over a multi-year period to analyze risks and trends in youth concussions. ${ }^{56}$ When comparing sports in which both boys and girls participated, the data suggested that girls had a higher rate of concussions than boys did. $^{57}$

However, there is no agreement that sex-based differences in TBI rates exist, and even if there were, it is not clear how such differences should inform youth sports TBI laws. Yet we know that the studies' inclusion of certain genderspecific sports like cheerleading (which is dominated by female athletes) significantly influences data trends and health solutions. When these sports are not included in such studies, it is likely that metrics will focus on comparisons between girls' and boys' divisions of common sports. As such, researchers who highlight the interaction between gender and TBIs would have the lawmaking process focus more on sex-specific youth sports TBI initiatives. These researchers argue against the sex-neutral interventions present in all youth sports TBI laws to date.

Despite evidence that sex-differentiation in TBI rates may exist, TBI research involving youth athletes focuses on areas with few to no cross-sex comparisons. These studies use different methods, lead to different conclusions, and sometimes contradict each other. For example, one recent high school sports study concluded that, holding other factors constant, there were no sex differences in the severity of concussions or short-term outcome measures, like recovery time. ${ }^{58}$ Some studies have found that female athletes have higher overall TBI rates,

55 See Gessel, supra note 23.

56 See Andrew E. Lincoln et al., Trends in Concussion Incidence in High School Sports: A Prospective 11-Year Study, 39 AM. J. SPORTS MED. 958 (2011) [hereinafter High School Trends Study].

$57 \mathrm{Id}$.

58 See Leah J. Frommer et al., Sex Differences in Concussion Symptoms of High School Athletes, $46 \mathrm{~J}$. ATHLETIC TRAining 76 (2011). 
while others suggest that such data may be due to heightened reporting of female TBIs. Broadly speaking, one cause of the heightened reporting could be a cultural bias in favor of being more protective of female athletes than their male counterparts. ${ }^{59}$ The same cultural biases may encourage male athletes to play even when they exhibit signs of concussions. Some boys suffering from head injuries may not report their symptoms for fear of being removed from play, which would depress male reporting even though overall rates might remain high. $^{60}$

While such available research might lean toward a more sex-nuanced approach to youth sports TBI laws, ${ }^{61}$ other evidence points toward a sex-neutral public health approach to TBIs. But there is one area of general agreement across the sex-based studies: boys' football dominates cases of TBIs, accounting for more than half of all reported youth TBIs. ${ }^{62}$ This rhetorical and scientific focus by commentators and policymakers on one particular sport's tendency to produce TBI events, in conjunction with existing anecdotal evidence of negative longterm consequences on professional football athletes, may be the motivating factor behind the football-based narratives underlying most (if not all) legislative discussions of youth sports TBI laws. On the other hand, such a focus in policymaking discussions could also indicate the NFL's influence on youth sports concussion policymaking.

Other studies suffer from similar data deficiencies when trying to parse population or incidence differences. For example, most studies of TBIs in youth sports do not account for non-scholastic athletes (athletes who play in recreational leagues or informal non-school community events), who tend to be younger and are often at even greater risk of concussion than youth athletes who play in school-sanctioned sports. Likewise, the data presented in such studies, by and large, does not differentiate between concussed athletes based on their socioeconomic or demographic information. ${ }^{63}$ Finally, while it is clear that those playing high-school football are the most susceptible to concussions, more study could provide information on whether athletes with certain physical

59 See, e.g., Patricia A. Vertinsky, The Eternally Wounded Woman: Women, Doctors, ANd EXercise IN THE LATE NineteEnTh Century 1-7 (1994).

60 See Mark R. Lovell et al., Inaccuracy of Symptom Reporting Following Concussion in Athletes, 34 MED. \& SCI. SPORTS \& EXERCISE, at S-298 (2002).

61 For example, calibrating return-to-play guidelines by sex if evidence shows that girls' injuries take longer to heal than boys' injuries, or calibrating symptom differentiation by sex to take into account whether boys and girls have higher baseline rates of common symptoms (like headaches) prior to concussions.

62 See Lincoln et al., supra note 56.

63 See Alan Schwarz, As Injuries Rise, Scant Oversight of Helmet Safety, N.Y. Times, Oct. 20, 2010, http://www.nytimes.com/2010/10/21/sports/football/21 helmets.html (describing fiscal constraints as one factor that school districts face when updating clearly outdated equipment intended to reduce injury risks). 
characteristics are more vulnerable to concussions.

Most studies that have analyzed youth populations have produced inconsistent results. However, several attempts have been made to apply a more rigorous analysis of the causes and consequences of sports-related brain injuries at the collegiate level, which can serve as a useful case study for youth athletics. The NCAA's comprehensive analysis ranks as one of the most authoritative studies of college sports to date (the "NCAA Study"). ${ }^{64}$ The NCAA Study focused on the recovery period after an athlete's initial injury and monitored both recovery time and the effects on an individual player following an on-field concussion. The study sought to bring a stronger empirical focus to determining when it is safe for a player to return to the game and the times during which a post-concussive player is most vulnerable to re-injury.

The NCAA Study evaluated the effects of sports-related concussions from the 1999-2001 football seasons. The NCAA studied 1631 football players from 15 NCAA Division I, II, and III institutions. ${ }^{65}$ Ninety-four players who were identified as having a football-related concussion were enrolled in an extensive injury-assessment protocol, and 70 of them completed the 90 -day protocol. The study's controls included a pre-season baseline evaluation with a health history questionnaire and a non-injured control team member. Players who suffered from a concussion ${ }^{66}$ were tested immediately after the injury, two to three hours after, and again on days $1,2,3,5,7$, and 90 post injury. The athletes underwent neuropsychology testing prior to the injury, as well as post injury on days 2,7 , and 90 .

The study revealed that concussions accounted for a significant percentage of total athlete injuries in a number of college sports: ice hockey $(12.2 \%)$, football $(8 \%)$, and soccer $(4.8 \%)$ in particular. Given the large number of program participants, college football had the highest overall number of brain injuries per year. The study stated that between 3 and $8 \%$ of hockey and football players sustain a concussion in each season, and the trends showed an increase during the years before the study's conclusion.

The study's most important finding was that by seven days after the injury,

64 See Michael McCrea et al., Acute Effects and Recovery Time Following Concussion in Collegiate Football Players: The NCAA Concussion Study, 290 JAMA 2556 (2003). The paragraphs that follow all reference this study.

65 The study was conducted before the recalibration and renaming of the divisions.

66 The study used the American Academy of Neurology Guideline for Management of Sports Concussion definition: "An injury resulting from a blow to the head causing an alteration in mental status and 1 or more of the following signs: Headache, nausea, vomiting, dizziness/balance problems, fatigue, difficulty sleeping, drowsiness, sensitivity to light or noise, blurred vision, memory difficulty, and difficulty concentrating." American Academy of Neurology, Practice Parameter: The Management of Concussion in Sports (Summary Statement): Report of the Quality Standards Committee, 48 Neurology 581 (1997). 
$91 \%$ of players had full resolution of symptoms. During that seven-day period, cognitive and verbal impairment persisted through at least two days, and milder cognitive effects persisted through five but often as long as seven days. Athletes had acute difficulties achieving balance during the 24 hours after injury but appeared to resolve these issues by the fifth day following the injury.

The NCAA Study also reinforced some now-conventional wisdom. The Study concluded that concussions can affect multiple domains (ionic, metabolic, and physiological) and can adversely affect core cerebral function for several days to weeks. In addition, the trajectories of recovery for these domains are not aligned. Symptoms, cognition, and balance recover at different speeds, complicating the process of determining when a player is safe to return to the field.

The NCAA Study's authors offered a few initial conclusions and recommendations. First, even after 90 days, players who experienced a concussion performed less well than control players did on verbal fluency measures. Second, college football players required, at a minimum, several days to recover after sports-related concussions. Third, cerebral dysfunction occurred in cases without typical indicators (like loss of consciousness and posttraumatic amnesia). Therefore, given the consequences of missing these subtle indications, medical professionals and standards-creating bodies should consider expanding or changing traditional definitions of concussion. Despite these strengths, the study failed to capture players who had the most "mild" forms of concussion, and the study's design was not fully able to identify factors that predict recovery across all areas or an appropriate duration of symptom-free recovery that would minimize risks of re-entry onto the playing field.

The High School Trends Study and the NCAA Study attempted to combine prior epidemiological studies into testing instruments and then evaluate extant knowledge against a sample of existing populations. Both studies, however, engaged in information gathering as much as hypothesis testing. To the extent that these two studies advanced prescriptive understandings of how regulatory bodies should approach incidences of TBIs, conclusions were sparse but clear: (a) TBIs are most prevalent in football with respect to overall incidences of TBIs, and a football-specific approach likely would achieve the most efficacious results; (b) incidences of low-grade concussions were much higher than previously estimated; (c) instances of low-grade concussions were fairly difficult to detect using traditional methods but easier to identify using baseline methodologies; and (d) instances of full recovery from TBIs took substantially longer than 24 hours.

Based upon these two prominent recent studies of TBIs in youth sports populations, one might expect that public health TBI interventions would: (a) focus on reducing the direct instances of TBIs, particularly with sport-specific solutions; (b) provide information-gathering and reporting mechanisms on the 
overall number of TBIs; (c) provide rigorous methods by which athletes, parents, and schools can detect subtle changes in athlete physiological characteristics; and (d) substantially extend recovery times by requiring lengthy "time-out" windows of five days or more following an athlete's suspected incurrence of a TBI. But the public health law interventions did not do any of the above. To understand how the policy prescription diverged from the medical evidence, the following section analyzes the component parts and provisions of all fifty youth sports TBI laws.

\section{RUSHING THE FIELD: YOUTH SPORTS TBI LAWMAKING (2009-2012)}

A study of the entire set of state youth sports TBI laws is a complex endeavor given the many causal inputs and policy variations. To provide the broadest and most accurate framing, this Part proceeds as follows. First, in Section III.A, the galvanizing incident prompting such laws will be described in its historical context. In Section III.B, a description of data gathering and methods will explain how these laws were analyzed and separated into their component parts. Next, Section III.C will examine common themes across states. Section III.D will analyze provisions that are only present in a minority of states but can be classified as "Lite-Experimental Policy" additions to a comprehensive youth sports TBI law. Section III.E will examine unique provisions in state laws that are of interest for further refinement of youth concussion laws and policies. Concluding the analysis, Section III.F will explore the common causes of policymaking choices during the legislative process, focusing on the role of the National Football League. The cumulative theory guiding each of these sections is that the NFL, through its unique role as a dominant interest group, established the content of states' youth sports TBI laws. The NFL's vigorous advocacy caused state legislatures to act swiftly, which minimized the role of scientific evidence and policy experimentation in the youth sports TBI lawmaking process.

\section{A. The "Galvanizing Incident" Model of Policymaking}

In 2006, Zackery Lystedt, then a 13-year-old middle-school student, sustained a severe head injury during a football game ${ }^{67}$ after he returned to play shortly after sustaining a TBI that was not properly evaluated. ${ }^{68}$ This post-TBI play led to additional head injury and eventually caused permanent brain

67 See Encouraging Signs for Teen Seriously Injured During Football Game, KомоNews.Com, Oct. 17, 2006, http://www.komonews.com/news/local/4422577.html.

68 Matt Rybaltowski, Young Player Helps Turn Trauma into Action on Concussions, CBSSPORTS.COM, Feb. 14, 2010, http://www.cbssports.com/nfl/story/12928497/young-playerhelps-turn-trauma-into-action-on-concussions. 
damage. ${ }^{69}$ Over the next several years, the Brain Injury Association of Washington worked with Lystedt's family to build a coalition of community, business, and sports organizations. ${ }^{70}$ This effort ultimately led the Washington State Legislature to unanimously pass the 2009 Zackery Lystedt Law, ${ }^{71}$ which set guidelines and standards to help recognize concussions and protect young athletes from immediate re-injury after sustaining an initial TBI. ${ }^{72}$ The statute was the first of its kind, specifically addressing TBIs in youth sports at the state level. ${ }^{73}$

To reduce concussion-related injuries among youth athletes, the Washington State law combined three essential elements that would become the basis for similar laws in other states. ${ }^{74}$ (1) The law required athletes, parents, and coaches to receive annual education sessions about recognizing the symptoms of concussions. If a young athlete is suspected of having a concussion, (2) he or she would be removed from a game or practice, and (3) would not permitted to return to play without clearance from a licensed healthcare professional. ${ }^{75}$ These provisions promoted injury recognition and post-injury care, two laudable public health goals. Nonetheless, they fell short of the full protections suggested by the epidemiological and empirical studies of TBIs discussed in Parts I and II of this Article. Most notably, the Zackery Lystedt Law did not try to prevent initial concussions within a public health law intervention framework. Further complicating matters, the Lystedt law defined the problem in a fairly tight bandwidth-the problem was "re-injury" and so the solutions focused attention on secondary prevention measures and avoided engaging primary prevention strategies as a legislative tool.

Following the enactment of the Zackery Lystedt Law, the NFL supported and engaged the issue by defining the problem narrowly. The NFL lobbied to have other states as well as the United States Congress follow suit and enact similarindeed, almost identical laws to the Lystedt Law. ${ }^{76}$ To advance this goal, NFL

69 Id.

$70 \mathrm{Id}$.

$71 \mathrm{Id}$.

72 The Zackery Lystedt Law, NFLEvolution.com, Aug. 9, 2012, http://www.nflevolution.com/article/The-Zackery-Lystedt-Law?ref $=270$ [hereinafter Zackery Lystedt Law].

73 This rapid response to one galvanizing incident may be part of a larger phenomenon of legislating by anecdote. See Glennon, supra note 51 , at 149 n.13 ("Legislative responses to highly unusual but extremely salient events often address issues of immediate public concern but typically ignore larger or more common structural issues."). Other examples include the Megan's Laws passed by various states, see, e.g., N.J. STAT. ANN. 2C:7-2, as well as the Terry Schiavo Law, Act for the Relief of the Parents of Theresa Marie Schiavo, Pub. L. No. 109-3, 119 Stat. 15 (2005).

74 Zackery Lystedt Law, supra note 72.

75 Id.

76 Id.; see H.R. Res. 6172, 11 th Cong. (2010). 
Commissioner Roger Goodell sent a letter to 44 states' governors urging legislation. ${ }^{77}$ Goodell also encouraged political and athletic leaders to help raise concussion awareness and promote proper post-concussive treatment. ${ }^{78}$ The NFL urged states to adopt the same three core elements as the Washington law, calling them the "three tenets of model legislation." ${ }^{, 79}$ However, during the process of encouraging the adoption of model legislation, the first tenet requiring parents and coaches to be annually educated "about the dangers of concussions" evolved to provide instead that parents or guardians of young athletes simply "sign a concussion information form" prior to allowing their child to engage in youth sports. ${ }^{81}$

The focus of legislative efforts on a more narrowly defined problem, following passage of the Lystedt Law was shaped, in part, by the NFL's early and visible involvement. Given this proactive effort by an interested and influential private for-profit interest group, it is not surprising that subsequent TBI legislation in many states exhibited remarkable uniformity based on the NFL's suggestions.

Defining the problem as re-injury allowed, as such definitions often do, for a consistent message and a default shaping of policy prescriptions. ${ }^{82}$ The role that the NFL played in promoting the Zackery Lystedt Law to other states as the basis for strikingly similar, if not identical, legislation can be viewed as a form of regulatory policy capture that effectively shaped the policy discussion and dwarfed policy input from other sources. ${ }^{83}$ While the laws will likely have some positive impact, the lawmaking process that created them may have unintentionally crowded out more innovative strategies. Although the scientific and analytic background for youth TBI policymaking is currently lacking, youth athletes are undoubtedly a population that deserves thoughtful, empirically grounded safety regulations that are rooted in a more expansive definition of the problem than allowed by the messaging of the NFL and others.

77 Concussion Legislation by State, NFLEvolution.COM, http://www.nflevolution.com/ article/Concussion-Legislation-by-State?ref $=767$ (last visited Feb. 9, 2013) [hereinafter Concussion Legislation]; see, e.g., Letter from Roger Goodell, Commissioner, Nat'l Football League, to Christopher Christie, Governor, N.J. (May 21, 2010), http://nflevolution.com/wordpress/wpcontent/uploads/2012/08/NJ-Gov-letter.pdf.

$78 \mathrm{Id}$.

79 Concussion Legislation, supra note 77.

80 See Letter from Roger Goodell, supra note 77.

81 Concussion Legislation, supra note 77.

82 See generally John W. Kingdon, Agendas, Alternatives, and Public Policies (2003).

83 See infra notes 123-124 and accompanying text. 


\section{B. Evaluating Youth Sports TBI Laws-Data and Methodology}

The current state of the field examined in this Article includes the youth sports TBI laws enacted by 49 states and the District of Columbia ${ }^{84}$ as of October 1,2013 . The state-by-state examination includes only the text of the law and does not include other provisions that might require additional actions of coaches or school districts, such as state agency administrative rules or district level policies. To facilitate future empirical evaluation of these laws, the legal research was conducted in accordance with scientific principles of transparency and reproducibility ${ }^{85}$ and is embodied in an open source protocol, codebook, and dataset available online at the Public Health Law Research Policy Surveillance Web Portal. ${ }^{86}$

The results of the legislative analysis are clear, identifying a high level of uniformity across state policies. This policy uniformity can best be assessed by comparing the clusters of common policy agreement among the laws and with reference to the Lystedt Law discussed earlier.

\section{A Uniform Policy Solution?}

When faced with a large public health problem, there are numerous policy options that might be employed with varying degrees of effectiveness. But with respect to TBIs in youth populations, the questions begin at a more basic level. What precisely is the problem? ${ }^{87}$ And why is public health law the place to solve it? Although there is consensus about the scope and cause of TBIs in adult populations, researchers, scientists, and advocates do not agree about the magnitude of youth TBIs or their root causes. ${ }^{88}$

Given a problem of such undetermined scope, whether public health law (rules, regulations, or other mandates) could influence an optimal public health

84 Mississippi is the only state without a youth sports TBI law as of October 1, 2013.

85 See, e.g., Jennifer K. Ibrahim et al., State Laws Restricting Driver Use of Mobile Communications Devices: Distracted Driving Provisions, 1992-2010, 40 AM. J. PrevenTIVE Med. 659,660-61 (2011) (describing a similar comprehensive statutory study).

86 See LAWATLAS, http://lawatlas.org (last visited Dec. 3, 2013).

87 If the problem were defined as youth having too many TBIs, preventive measures would likely be more invasive (e.g., banning certain sports or activities). If the problem is schools failing to enact simple risk-reduction measures, law might provide for simple mandates. Or, if the problem is that cultural norms prevent more invasive sport-specific measures, perhaps the solution might involve education about sport-specific dangers in an attempt to proactively shape public opinion.

88 See, e.g., Nat'l Ctr. for Injury Prevention \& Control, Traumatic Brain Injury in the United States: Assessing Outcomes in Children, Centers for Disease CONTROL \& Prevention (2000), http://www.cdc.gov/traumaticbraininjury/pdf/TBI_assessing.pdf (noting that "TBI is often described as the leading cause of disability in children, but data to support this assertion are lacking .... Currently no population-based studies of the outcomes of TBI among children and youth exist to provide national estimates of TBI-related disability and document the need for services"). 
outcome (reducing youth sports TBIs and their health consequences) was not clear at the outset of the proposed policy solutions. ${ }^{89}$ Therefore, although public health advocates and health professionals have long known that TBIs and MTBIs may lead to short- and long-term health consequences, ${ }^{90}$ no public health law interventions were developed in response to the growing awareness of such problems in youth populations prior to 2009. However, the confluence of the events described earlier, a growing consciousness of health risks among college and professional athletes, numerous tragic professional athlete deaths, and the motivation of a powerful outside actor (the NFL) created an environment ripe for state legislative action. ${ }^{91}$

Between 2009 and 2013, 49 states and the District of Columbia enacted one or more youth sports TBI laws. There are no states that have banned traditional youth sports with high TBI risks or that have set out legal regimes attempting to govern particular sports techniques by legislation or regulatory oversight.

In concert with the public advocacy of the $\mathrm{NFL}^{92}$ and with the inclusion of the three tenets of Washington's Lystedt Law, ${ }^{93}$ many of the other states' legislation includes all three tenets in one form or another. As previously stated, the laws require: (1) that a youth athlete who appears to have suffered a concussion be removed from play or practice at the time of the suspected concussion for a minimum of 24 hours, ${ }^{94}(2)$ that a youth athlete be cleared by a

89 Some intervention, of course, would coalesce on the minimum that we do know, such as the consensus that it is generally harmful to have multiple TBIs and certainly within a short period. Nonetheless, if the overall optimal goal is to generally reduce TBIs in the first instance, the epidemiology is less clear.

90 Various advocacy and trade groups set out detailed concussion prevention proposals many years ago, and the CDC has provided detailed information and guidelines for more than a decade. See, e.g., Traumatic Brain Injury: A Case for Prevention, Mass. Traumatic Brain Injury PREVENTION TASK FORCE (2007), http://www.mass.gov/eohhs/docs/dph/com-health/injury/tbi-caseprevention.pdf (report convened by the Massachusetts Department of Public Health detailing existing state TBI data and recommending prevention strategies, including surveillance and evaluation, policy and enforcement, and education and training).

91 See Alan Schwarz, N.F.L. Players Shaken by Duerson's Suicide Message, N.Y. TimeS, Feb. 20, 2011, http://www.nytimes.com/2011/02/21/sports/football/21duerson.html.

92 See Concussion Legislation, supra note 77.

93 See Zackery Lystedt Law, WASH. REv. CODE $§ 28$ A.600.190 (2013).

94 But there is not scientific agreement that the 24-hour return-to-play minimum is optimal (as opposed to a longer minimum rest), nor is there agreement for how medical professionals might design an optimal period of recovery. See Paul McCrory et al., Consensus Statement on Concussion in Sport: The 4th International Conference on Concussion in Sport Held in Zurich. November 2012, 47 BRIT. J. SPORTS MED. 250 (2013). It may be that the focus on a 24-hour minimum in the NFL's messaging, contrary to most scientific studies, is one example of the impact such messaging had in the universal adoption of the 24-hour minimum as opposed to a longer, more supported waiting period. 
licensed health care professional trained in the evaluation and management of concussions before returning to play or practice ${ }^{95}$ and (3) that each year, athletes, parents, and coaches receive education or a information sheet about recognizing the symptoms of concussions. ${ }^{96}$ Thus, key features across all youth sports TBI laws include a focus on secondary, not primary prevention, as well as a general adherence to the Lystedt framework with minimal policy experimentation. ${ }^{97}$ In Table 1 on the following page, state laws are compared by reference to their most common elements and those of the Lystedt framework. ${ }^{98}$

95 See Concussion Legislation, supra note 77.

96 See id:; Letter from Roger Goodell, supra note 77.

97 There are other explanations for states following the Lystedt model (and subsequent state frameworks based on this model). A behavioral critique of lawmakers might suggest that such lawmakers often follow the status quo or default policy position on public health issues and take the path of least resistance when evaluating a new policy measure in the face of an existing public health policy already promulgated in another jurisdiction. See, e.g., Eric J. Johnson \& Daniel Goldstein, Do Defaults Save Lives?, 302 SCIENCE 1338 (2003).

98 This table does not represent all of the variables, and statutes that have been modified since enactment are not fully incorporated. 
Refereeing the Public HeAlth

\begin{tabular}{|c|c|c|c|c|c|c|c|c|c|c|c|c|c|}
\hline \multirow[b]{3}{*}{ STATE } & \multicolumn{13}{|c|}{ Table 1. Key Features of Youth S ports TBI Laws (2009 - 2013) } \\
\hline & \multirow[b]{2}{*}{$\begin{array}{c}\text { Has Youth } \\
\text { Sports TBI } \\
\text { Law }\end{array}$} & \multirow[b]{2}{*}{$\begin{array}{c}\text { Requires } \\
\text { Removal } \\
\text { From Play }\end{array}$} & \multirow[b]{2}{*}{$\begin{array}{c}\text { Requires } \\
\text { Parental } \\
\text { Notificatio } \\
\mathbf{n}\end{array}$} & \multicolumn{5}{|c|}{ Return to Play Requirements } & \multirow{2}{*}{\begin{tabular}{|l|} 
Training \\
\\
\\
Requires \\
Special \\
Training for \\
Coaches \\
\end{tabular}} & \multicolumn{4}{|c|}{ TBl Information/ $\Lambda$ wareness Requirements } \\
\hline & & & & $\begin{array}{c}\text { Specifies } \\
\text { Retum To } \\
\text { Play } \\
\end{array}$ & \begin{tabular}{|c|} 
Requires \\
Clearance \\
by a Health \\
Professiona \\
I \\
\end{tabular} & $\begin{array}{c}\text { Requires } \\
\text { Written } \\
\text { Clearance }\end{array}$ & \begin{tabular}{|c|} 
Health \\
Professiona \\
$1 \mathrm{~T}$ Trained in \\
TBI \\
Manageme \\
nt Required \\
\end{tabular} & $\begin{array}{c}\text { Clearance } \\
\text { by a } \\
\text { Medical } \\
\text { Doctor } \\
\text { Required } \\
\end{array}$ & & \begin{tabular}{|c} 
Requires \\
Information \\
Sheet \\
Distributio \\
$n$ \\
\end{tabular} & $\begin{array}{l}\text { Parent's } \\
\text { Signat ure } \\
\text { Required } \\
\end{array}$ & $\begin{array}{l}\text { Student's } \\
\text { Signature } \\
\text { Required }\end{array}$ & $\begin{array}{c}\text { Information } \\
\text { Sheel } \\
\text { Distributed } \\
\text { on an } \\
\text { Annual } \\
\text { Basis } \\
\end{array}$ \\
\hline $\mathrm{AK}$ & 1 & 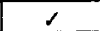 & & $\checkmark$ & 10 & 1 & 1 & & & 1 & & 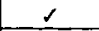 & $\checkmark$ \\
\hline$\overline{A L}$ & 1 & 7 & & $\checkmark$ & 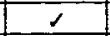 & $r$ & & 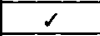 & $\checkmark$ & 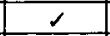 & 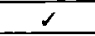 & $r$ & 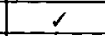 \\
\hline AR & $\overline{1}$ & 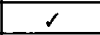 & & $\checkmark$ & $\checkmark$ & 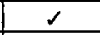 & $\checkmark$ & & & 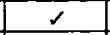 & & $\underline{r}$ & 1 \\
\hline$A Z$ & $\checkmark$ & 1 & & $\alpha$ & 1 & 1 & 1 & & & 1 & 1 & 1 & 1 \\
\hline $\mathrm{CA}$ & 1 & 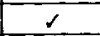 & & $\therefore$ & 1 & 1 & $\therefore$ & & $\therefore$ & $r$ & 1 & 1 & 1 \\
\hline $\mathrm{CO}$ & 1 & 1 & 1 & $\checkmark$ & 1 & $\sigma$ & 5 & & $\sigma$ & & & & \\
\hline CT & $r$ & 1 & & 1 & 1 & 6 & $\checkmark$ & & $\checkmark$ & & & & \\
\hline $\mathrm{DC}$ & 8 & 1 & & $\checkmark$ & 1 & 1 & & & & $\checkmark$ & $\checkmark$ & $\checkmark$ & \\
\hline $\overrightarrow{D E}$ & $\therefore$ & 1 & & $\sigma$ & 1 & 1 & 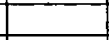 & 1 & 1 & $\therefore$ & $\therefore$ & 1 & 1 \\
\hline $\mathrm{FL}$ & $\checkmark$ & 1 & & $\angle$ & $\checkmark$ & 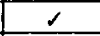 & 1 & & & 1 & 1 & & $\checkmark$ \\
\hline $\mathrm{GA}$ & 8 & 1 & & $\checkmark$ & 1 & & 1 & & & $\checkmark$ & & & 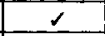 \\
\hline $\mathrm{HI}$ & $\checkmark$ & 1 & & $\checkmark$ & $\therefore$ & 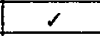 & 1 & & 1 & 1 & 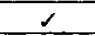 & 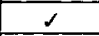 & 1 \\
\hline $\mathrm{IA}$ & 1 & 1 & & 1 & 1 & 1 & $\checkmark$ & & & 1 & 1 & 1 & 1 \\
\hline ID & $\therefore$ & $\checkmark$ & & 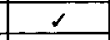 & 1 & & 1 & & & 1 & & & 1 \\
\hline $\mathrm{IL}^{*}$ & 1 & - & & - & & & & & & & & & \\
\hline $1 \mathrm{~N}$ & $\checkmark$ & $\therefore$ & & 1 & $\therefore$ & $\checkmark$ & $\checkmark$ & & & $\checkmark$ & 6 & 7 & $\sigma$ \\
\hline $\mathrm{KS}$ & 1 & $\checkmark$ & & $\checkmark$ & 1 & 1 & & 1 & & $\checkmark$ & 1 & $\checkmark$ & 1 \\
\hline $\mathrm{KY}$ & 1 & $\therefore$ & & 1 & $\therefore$ & 1 & 1 & & $\checkmark$ & & & & \\
\hline$\overline{L A}$ & 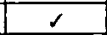 & 1 & 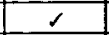 & $\checkmark$ & $\sigma$ & 1 & 1 & & $\checkmark$ & .8 & 8 & 1 & $\therefore$ \\
\hline MA & 1 & 8 & & $\checkmark$ & $\sigma$ & 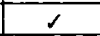 & 1 & & 6 & $\sigma$ & & & \\
\hline MD & 1 & 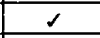 & & $\checkmark$ & $\checkmark$ & 1 & 1 & & - & $\therefore$ & $\sigma$ & $r$ & \\
\hline $\mathrm{ME}^{*}$ & 1 & & & & & & & & & & & & \\
\hline MI & 1 & 1 & & 1 & $\checkmark$ & 1 & 1 & & & 1 & $\gamma$ & 1 & \\
\hline MN & $\therefore$ & 1 & & $r$ & 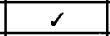 & 1 & $\therefore$ & & $\therefore$ & & & & \\
\hline MO & 1 & $\checkmark$ & & 1 & 1 & $r$ & 1 & & & 1 & $\sigma$ & & $\checkmark$ \\
\hline \multicolumn{14}{|l|}{$\mathrm{MS}^{* *}$} \\
\hline MT & $\checkmark$ & 6 & & 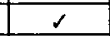 & $\checkmark$ & 8 & 1 & & 1 & $\checkmark$ & 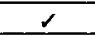 & 1 & 1 \\
\hline NC & 1 & $\therefore$ & & $\checkmark$ & 1 & 1 & & & & 1 & 1 & 1 & 1 \\
\hline ND & 1 & $\checkmark$ & & $\checkmark$ & 1 & $\checkmark$ & 1 & & $\checkmark$ & $r$ & 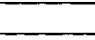 & & \\
\hline $\mathrm{NE}$ & $\checkmark$ & 1 & $r$ & 1 & 1 & 1 & 1 & & & $r$ & & 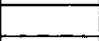 & $\checkmark$ \\
\hline NH & $\checkmark$ & 1 & $\checkmark$ & $\therefore$ & 1 & 1 & 1 & & & & & & \\
\hline $\mathrm{NJ}$ & 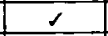 & 8 & & 1 & 1 & 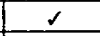 & 1 & 1 & 1 & 1 & 1 & 1 & $\checkmark$ \\
\hline NM & 1 & 1 & & $\checkmark$ & 1 & & & & 1 & $\therefore$ & $\therefore$ & 1 & $\alpha$ \\
\hline $\mathrm{NV}$ & $\checkmark$ & 1 & & 1 & $\checkmark$ & 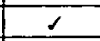 & & & & $\checkmark$ & $\sigma$ & 1 & $\checkmark$ \\
\hline NY & 1 & 1 & & 1 & 1 & $\therefore$ & & $\delta$ & $\checkmark$ & 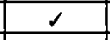 & & $\ldots$ & $\ldots$ \\
\hline $\mathrm{OH}$ & 1 & 1 & & 1 & 1 & $\gamma$ & & & $\checkmark$ & $\therefore$ & $\therefore$ & & 1 \\
\hline $\mathrm{OK}$ & 1 & 1 & & 1 & 1 & $\checkmark$ & 1 & & & $\therefore$ & $\sigma$ & 1 & $\checkmark$ \\
\hline $\mathrm{OR}$ & $\checkmark$ & $\therefore$ & & 1 & 1 & $\therefore$ & & & 1 & & & & \\
\hline $\mathrm{PA}$ & 1 & $\checkmark$ & & 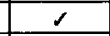 & 1 & $\sigma$ & 1 & & $\sigma$ & $\checkmark$ & $\checkmark$ & 1 & 1 \\
\hline $\mathrm{RI}$ & 1 & $\checkmark$ & 1 & 1 & 8 & 8 & 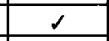 & 1 & 1 & 1 & 1 & & $\checkmark$ \\
\hline$s c$ & 1 & 1 & & 1 & 1 & 1 & & $r$ & & 1 & $\gamma$ & & 1 \\
\hline SD & 1 & 6 & & 1 & 1 & 1 & 1 & & 1 & 1 & 1 & 1 & 1 \\
\hline $\mathrm{TN}$ & 1 & 1 & & 1 & $\sigma$ & 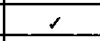 & & & 1 & z & & & 1 \\
\hline$T X$ & 1 & $\checkmark$ & $\checkmark$ & $\checkmark$ & $r$ & $\checkmark$ & & $\checkmark$ & $\checkmark$ & 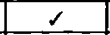 & $r$ & 1 & 6 \\
\hline UT & 1 & $\overline{1}$ & & $\checkmark$ & 1 & 1 & 1 & & & 1 & $\therefore$ & & \\
\hline$\overline{V_{A}}$ & $\sigma$ & 2 & & 1 & 1 & $\therefore$ & & & & 1 & 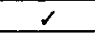 & 1 & 1 \\
\hline $\mathrm{VT}$ & $\sigma$ & $\vec{s}$ & 1 & 1 & 8 & 1 & 1 & & $\sigma$ & 1 & $\checkmark$ & 8 & $\checkmark$ \\
\hline WA & $\therefore$ & $\therefore$ & & $\therefore$ & $\checkmark$ & 1 & 6 & & & 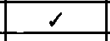 & 1 & 1 & 1 \\
\hline$w_{1}$ & 1 & 1 & & 1 & $\checkmark$ & 1 & $\checkmark$ & & & 1 & 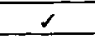 & 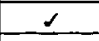 & 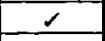 \\
\hline wV & 1 & $r$ & & 1 & 1 & 1 & 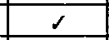 & & 1 & 1 & 1 & $\checkmark$ & $\checkmark$ \\
\hline WY & 1 & & & & & & & & $\checkmark$ & & & & \\
\hline Total & 50 & 47 & 7 & 47 & 47 & 44 & 33 & 8 & 25 & 41 & 32 & 28 & 34 \\
\hline
\end{tabular}


Most states follow the first Lystedt tenet-removal from play following an actual or suspected TBI. Forty-six states and the District of Columbia have such a requirement, and all but Wisconsin and Ohio require removal for a minimum of 24 hours. The states that have such a minimum time length removal requirement do not substantively vary in their approach to re-entry. No state law mandates a minimum removal time beyond 24 hours, although the accompanying health evaluation and clearance provisions (discussed below) may effectively create a longer minimum removal provision by default.

The states differ much more on the second tenet of the model legislation, which requires that athletes with concussions or TBIs be cleared by a medical or health professional before return to play. Forty-four of the 50 jurisdictions with youth sports TBI laws have provisions requiring written clearance, but the type of medical professional required to conduct the evaluation varies widely by state. Only eight states require that a medical doctor provide the clearance. Some require that such professionals must be medical specialists of some kind, such as licensed physicians (with or without TBI training). Still other states permit neuropsychologists to provide clearance. Athletic trainers and nurses (who can also be specialists in many areas) are included in some clearance provisions but not others. For example, some suggest that athletic trainers could have a conflict of interest, especially in instances where the player's return to the active roster would benefit the team. However, whether this potential conflict of interest influences clearance decisions in practice is unknown. ${ }^{99}$ Perhaps responding to ambivalence about laws that allow non-specialist physicians to provide clearance, Rhode Island eventually introduced legislation to amend its youth concussion policy to require clearance from a licensed healthcare professional "trained in the evaluation and management of concussions" rather than by a physician. ${ }^{100}$ This focus on all providers having TBI training eventually emerged as a new majority position. Thirty-two jurisdictions require that a clearance provider be specifically trained in TBI recognition and/or symptom management. Why the remaining jurisdictions do not is unclear. But generally speaking, this example of divergence in policymaking with respect to one portion of one provision

99 See, e.g., Press Release, Am. Acad. of Pediatrics, High Schools with Athletic Trainers Have More Diagnosed Concussions, Fewer Overall Injuries (Oct. 22, 2012), http://www.aap.org/en-us/about-the-aap/aap-press-room/pages/High-Schools-with-Athletic-

Trainers-have-More-Diagnosed-Concussions-Fewer-Overall-Injuries.aspx.

100 R.I. GEN. Laws § 16-91-3 (2013); Telephone Interview with Rep. Raymond E. Gallison Jr., Rhode Island House of Representatives (June 20, 2011) (noting that Rhode Island Interscholastic League, through its sports medicine subcommittee, brought forth recommendations to Rep. Gallison advising that athletes who suffered or were suspected of suffering concussions should only be evaluated by specialists in concussion management). 
demonstrates a lack of general scientific (and political) consensus about why a particular health professional should perform this clearance function or whether one type of professional is preferable to another.

With respect to the third tenet, 41 states and the District of Columbia require that some form of TBI information be distributed to parents and student athletes, yet only 34 of these jurisdictions require distribution on an annual basis. Parents or guardians must provide a signature release that they have received the information. The substance of such education and the form's language are not specified in the laws, although the Centers for Disease Control and Prevention's (CDC) well-disseminated materials have been explicitly mentioned in some laws as an initial guidepost for creating such educational materials.

Many states have innovated beyond the three tenets while staying well within their spirit. Twenty-five jurisdictions require additional coach education for recognizing the symptoms of TBIs in youth sports. However, the additional education requirements substantially vary. Some states, such as Vermont and New York, require coaches to receive training every two years, ${ }^{101}$ while Arkansas requires that coaches receive training every three years. ${ }^{102}$ Although a few states, such as Michigan, provide general guidelines regarding the broad contours of a training program or methods of distributing training materials, none of the laws studied here define exactly how such coach education efforts should be constructed. The laws delegate the task of determining the educational programs' content to third parties such as school boards, athletic associations, or departments of health. However, in many of these states, coaches may be subject to concussion education requirements mandated by their voluntary membership associations or athletic governing bodies, which can be more rigorous than the state-mandated requirements. There is no compelling reason why states should avoid training requirements in youth sports TBI laws, given the wide availability of information and its low cost. ${ }^{103}$

States also share a common understanding that the implementation of their youth sports TBI laws should be handled collaboratively with key stakeholders. Most states designate one or several statewide groups to develop policies and standards for youth concussion awareness, as well as protocols that may be adopted by individual school districts and athletic programs. The groups creating the guidelines are often the state departments of education and/or health; ${ }^{104}$ however, some states require other types of organizations, both public and

101 See N.Y. Educ. LaW $\S 305$ (McKinney 2013); VT. STAT. ANN. tit. 16, § 563 (2013).

102 See ARK. CODE ANN. § 6-18-708 (2013).

103 See, e.g., Concussion in Sports: What You Need to Know, NFHS Learning CENTER, http://www.nfhslearn.com/electiveDetail.aspx?courseID $=38000$ (last visited Dec. 5, 2013).

104 See, e.g., MD. Code AnN., Educ. § 7-433 (West 2013); N.Y. Educ. Law $\S 305$ (McKinney 2013); 24 PA. Cons. STAT. $\S 5323$ (2013). 
private, to coordinate and develop youth concussion guidelines. For example, Idaho requires the State Board of Education to work in collaboration with the Idaho School Activities Association to "provide access to appropriate guidelines and information that identify the signs and symptoms of a concussion and head injury." ${ }^{105}$ Similarly, South Dakota specifies that the state's high school athletic conference and its Department of Education "shall develop guidelines to inform and educate member schools, coaches, athletes, and the parents or guardians of athletes, of the nature and risk of concussion."106 Presumably, these guidelines are created by those who have the resources and expertise to provide local districts with scientifically accurate and current information. This type of localized information gathering and policy specification could be more efficient compared to a requirement that each district create separate, and perhaps incomplete, youth concussion policies.

This policy innovation with respect to educating key constituents (e.g., coaches, parents, youth athletes, schools) comes with some scientific uncertainty about the link between constituent education and the policy goal of such education-reducing youth sports TBIs. For example, although the CDC has created broad youth sports TBI guidance, the fidelity of state and local informational materials to CDC guidance is currently unknown.

Therefore, the particulars of the coach and parent "education" components of these laws present a number of important issues for implementers and researchers. First, the efficacy of education and consent in helping parents or coaches prevent, identify, and respond to TBIs in this context is unknown. Second, the content of the required education is in most cases not specified in the laws - thus allowing for wide variance in implementation. Third, there is divergence between the education regimes required for key stakeholders (e.g., parents vs. coaches), which may produce inconsistent responses to potential TBI events. Fourth, as some training materials already exist, a system in which each state crafts its own standards from scratch may be inefficient. Finally, some coaches may already receive substantial training through membership athletic associations and other non-government entities, but the laws do not contemplate the integration of such education into a more uniform education approach among stakeholders such as coaches, parents, athletes, and schools.

Overall, the above concerns are examples of the limitations of relying on the Lystedt's limited framework. The Lystedt framework, which does not provide detailed guidance for how coaches and parents should be educated, thus allows for experimentation and ambiguity at the expense of policymaking clarity through a centralized framework. Further, because the Lystedt framework provides only general guidance, states could have pushed beyond its provisions

105 See IDAHo CODE ANN. § 33-1625 (2013).

106 S.D. CoDifiED LAWS $\S 13-36-9$ (West 2013). 
when creating their own laws. Some states did take such an incrementally experimental approach in an effort to more aggressively solve the problem and innovate outside of the Lystedt framework, and to such experimentation we now turn.

\section{The Role of Policy Experimentation (Purposeful and Accidental)}

One example of a policy innovation adopted by a small minority of states (and likely consistent with sound science) is the requirement that states review and update their state youth concussion information sheets to determine the program's effectiveness and make revisions as new techniques to identify and treat concussions become available. Without evaluative metrics and mandatory state-level reporting built into the framework, however, how will the states know how to update their policies? If the states with annual review criteria have no way to analyze results from the field or assess whether the legislation is producing its desired effect, it will be difficult to successfully apply a logic/feedback model to improve existing information.

Defining the scope of "athletic activity" may have resulted in a form of accidental policy experimentation. Many states failed to broadly define youth athletic activity, thus narrowing the scope of the youth sports covered under existing laws. Specifically, the majority of youth sports TBI laws do not include non-scholastic athletic team activity, such as athletic clubs and leagues that are not affiliated with a school or school district. In addition, Utah is one of the handful of states where the youth concussion policy specifically includes physical education, ${ }^{107}$ an athletic activity in which more than half of high-school students in the United States participate. ${ }^{108}$ Furthermore, few chose to include programs or teams associated with county recreation departments in their youth concussion guidelines. While there may be a higher prevalence of concussions among athletes participating in organized scholastic sports such as football, lacrosse, or soccer, ${ }^{109}$ this does not mean that children participating in other activities such as physical education class could not benefit from coverage by provisions in youth sports TBI legislation. ${ }^{110}$ The states that have not yet adopted

107 UTAH CODE ANN. § 26-53-401 (West 2013).

108 Ctr. for Disease Control \& Prevention, Youth Risk Behavior Surveillance-United States, 2011, MORBIDITY \& MORTALITY WKLY. REP., June 8, 2012, at 37.

109 See Lincoln et al., supra note 56. Football accounts for more than half of concussions, while baseball had the lowest incidence of concussion. In girls' sports, soccer represented the highest proportion of concussions, followed by cheerleading, basketball, and lacrosse. Overall concussion rates for boys are more than double those for girls. Id.

110 Youth Concussion FAQ, NFL, http://www.nfl.com/news/story/0ap1000000228346/ article/youth-concussion-faq (last updated Aug. 9, 2013) ("Concussions can occur in athletes of any age and in any sport or recreational activity. In fact, each year, U.S. emergency departments treat 
such policies (and even those that have) could consider broadening their definition of athletic activity to be more inclusive.

Nonetheless, even if these laws do not expansively cover all forms of youth athletics, during the implementation of youth concussion policies, individual school districts could voluntarily apply concussion training to physical education courses and students. Some states even have language in their youth sports TBI laws encouraging youth sports programs not included in the legislation to adopt a similar policy ${ }^{111}$ or requiring those organizations that use school facilities to comply with the youth concussion policy. ${ }^{112}$ While it is certainly possible that schools or youth concussion policymakers may be able to apply safety policies to athletic activities not covered by the law, the lack of a specific provision in such laws means that children participating in non-scholastic athletic teams may receive a lower standard of care if they suffer a TBI.

\section{E. Substantive Policy Innovation: Evidence, Theory, and Practice}

In addition to the three tenets discussed in Section III.C and other policy experimentations discussed in Section III.D, a few states have developed novel provisions that could be the basis for future model legislation. The role of baseline testing provides one such area for experimentation. Rhode Island's youth sports TBI law previously contained a provision that "[s]chool districts are encouraged to have all student athletes baseline or [ImPACT] tested prior to the start of every sport season." "13 Subsequently, Rhode Island passed legislation to remove the ImPACT testing requirement and substitute the test with a free, nonproprietary evaluation tool. ${ }^{114}$ This type of provision could prove to be more effective in actually detecting and treating TBIs among young athletes than simple TBI identification training. However, the administration of such a test for large groups of young athletes may have an added cost for school districts and parents.

an estimated 135,000 sports-related and recreation-related TBIs, [i]ncluding concussions, among children ages 5 to $18 . ")$.

111 See, e.g., R.I. GEN. LAws $\S 16-91-4$ (2013) ("All other youth sports programs not specifically addressed by this statute are encouraged to follow the guidance set forth in this statute for all program participants who are age nineteen (19) and younger.").

112 See ARIZ. REV. STAT. ANN. $\$ 15-341$ (2013) ("A group or organization that uses property or facilities owned or operated by a school district for athletic activities shall comply with the requirements of [the youth concussion policy]").

113 R.I. GEN. LAwS § 16-91-3 (2010).

114 See 2010 R.I. Pub. Laws. 112 (codified as amended at R.I. GEN. Laws ANN. § 16-91-3 (West 2013)); Telephone Interview with Rep. Raymond E. Gallison Jr., Rhode Island House of Representatives (June 20,2011) (stating that the Rhode Island Interscholastic League, the agency to which all high-school athletic teams belong, sought to remove the baseline testing provision of the law since it was a proprietary test; at the time of the interview, a substitute baseline testing was being developed by subcommittee for free use). 
The NCAA Study and many others have concluded that proper baseline and psychological testing, coupled with an extensive post-injury rest period, is most likely to help properly identify and mitigate re-injury. Nonetheless, there is not yet professional consensus regarding the best standard diagnostic procedure or metric for TBIs or the professional qualifications required to make the decision that an athlete should be able to return to play. Should agreement on a best TBI diagnostic metric or clinical best practice emerge, legislatures may need to amend their statutes to adopt the proven practice, including the appropriate professional qualifications for those who provide clearance. Thus, Rhode Island's policy experimentation will provide useful data metrics, test a novel populationimpacting approach to harm reduction, and clarify areas of scientific ambiguity. ${ }^{115}$

Another policy innovation adopted by two states imposes harsh penalties on coaches who fail to comply with the youth sports TBI law provisions. In Pennsylvania, the governing body of a school is required to suspend a coach for the remainder of a season if the coach fails to properly remove a student athlete from play. ${ }^{116}$ For a subsequent violation, a coach can have a longer suspension or even a "permanent suspension from coaching any athletic activity." 117 Similarly, in Connecticut, the State Board of Education may revoke a coaching permit for a coach who fails to remove a student suspected of having a concussion from play. ${ }^{118}$ A coaching permit can also be revoked for a Connecticut coach's failure to complete training and refresher courses on concussion recognition and treatment. ${ }^{119}$ These types of provisions should help remove any sort of incentive that a coach might have to play a young athlete when the athlete has suffered a TBI, but few states have adopted these provisions. The reasons for most states' failure to consider such innovations are unclear.

Finally, states differ in the degree to which their youth sports TBI laws insulate various constituents from civil liability. Twenty-five jurisdictions have enacted youth sports TBI laws that attempt-directly or by inference-to limit liability for school districts, volunteers, healthcare providers, and others who might face lawsuits filed by athletes, their families, and others for damages on the basis of how these individuals respond to TBI-related events. These provisions usually have a carve-out for gross negligence and willful or wanton

115 This is precisely the type of policy innovation that, with respect to other statewide public health law matters, one might hope that the state policy laboratories would allow to flourish. However, as discussed earlier, the strong uniformity among state laws has made the "can states act as policy laboratories?" approach to lawmaking with respect to TBIs a rhetorical question.

11624 Pa. Cons. STAT. $\$ 5323$ (2013).

117 Id.

118 ConN. Gen. Stat. § 10-149c (2013).

119 Id. 
misconduct. ${ }^{120}$ A typical provision provides that a protected party "shall be immune from civil liability for good faith conduct arising from or pertaining to the injury or death of a student-athlete" if the party provides some proof that the conduct was in compliance with the law and "local school board policies relative to the management of concussions and head injuries."121 Other statutes are more ambiguous, stating that the law does not create a "new cause of action" but not specifying whether other portions of state law might still provide for civil liability.

In contrast, the other twenty-five jurisdictions with youth sports TBI laws have determined (whether by silence or legislative debate) that the duties created by their youth sports TBI laws do not warrant special discussion of potential liability for key actors or immunity from civil liability. This decision to not provide waivers may have stemmed from the lack of evidence assessing whether liability waivers positively or negatively influence the identification and management of TBIs. This patchwork of liability waivers among types of parties and across states raises novel legal questions. ${ }^{122}$ For example, contests may involve athletes from different states or take place outside of one state's jurisdiction under a different set of liability rules. In those circumstances, which state's provisions will apply, and how do we know in advance?

\section{F. Evidence, Influence, and Rushing: Common Causal Mechanisms in Youth Sports TBI Law Policymaking}

While the specific motivation for youth sports TBI laws may be different in every state, there appear to be three common elements that motivated state lawmakers during the period studied here. Ideally, proposed legislation would be motivated solely by a broad set of public health law goals upon which all agreed, although realistically moderated by concerns such as fiscal constraint. However, for reasons explained earlier, the logic of youth sports TBI laws lacks a tight alignment with most scientific studies of the root causes and treatments of youth sports TBIs. This lack of alignment may be due to the actual motivating elements of the legislation, which have generally been threefold: the lobbying effort of the NFL, the sudden national attention given to the rise in youth concussions, and the desire to minimize the cost in implementing new state regulations covering a large portion of a state's population and infrastructure. The combination of these

120 See, e.g., IND. CODE $\S 20-34-7-5$ (2013).

121 N.H. Rev. StaT. ANN. § 200:51 (2013).

122 The connection between liability waivers and broad public policymaking on public health and other matters is a subject worthy of its own detailed analysis and cannot be fully explored here. However, it is certainly true that the relationship between waivers and policy efficacy has not been adequately studied, and one cannot be certain that such provisions will help lower the overall baseline TBI rates. 
three elements provides one broad explanation for the rapid adoption of virtually identical laws across forty-nine jurisdictions. Each motivating element shall be examined in turn.

With respect to the NFL's role, public choice scholars have commented extensively on outside interest groups' roles in influencing state policymaking. Here, the states' legislative actions could be considered a form of regulatory capture. ${ }^{123}$ In short, regulatory capture occurs when special interests compete to use government power for their own agenda. ${ }^{124}$ Under the encompassing theory of public choice, government policymakers act for their constituents, as the "public," in creating an outcome, the "choice." 125 The outcome of a regulatory capture will represent the interest groups' agenda and (potentially) a form of government policymaking failure. ${ }^{126}$ Obviously, this outcome is not always best for the public interest. ${ }^{127}$

State-level lawmaking with respect to youth sports TBI laws suggests capture, though other alternatives are also plausible. In combination with the previously discussed absence of empirical evidence on which to base their policymaking, the states' legislative efforts could be captured-whether in rhetoric, strategic focus, or policy-by one powerful private interest group that was ready and willing to lead the charge. Therefore, if the policy "choice" has been captured, we should be concerned about whether this choice truly is the public will. That the NFL vigorously supported states' policymaking against an empirically ambiguous backdrop also raises concerns about how the resulting laws will operate and whether they will optimally benefit youth athletes. The laws may well prove to have a positive impact, and the NFL's role should then be commended. However, for reasons described earlier, the absence of evidence and data metrics suggests that it will be hard to determine in the short term what the impact has been.

The evidence gathered for this Article suggests that the NFL stepped into a policy vacuum with a compelling legislative message. This messaging built on the momentum generated by the events leading up to the Washington State Lystedt Law and ultimately shaped the policy debate from 2009 to present. To be fair, with any complex story, there are multiple causal explanations, and many of them deserve their own separate analysis. For example, it could be that states

123 See generally Sam Peltzman, Toward a More General Theory of Regulation, 19 J.L. \& ECON. 211 (1976); Richard Posner, Theories of Economic Regulation, 5 BELL J. ECON. \& MGMT. SCI. 335 (1974); George Stigler, The Theory of Economic Regulation, 2 BELL J. ECON. \& MGMT. SCI. 3 (1971).

124 Michael E. Levine \& Jennifer L. Forrence, Regulatory Capture, Public Interest, and the Public Agenda: Toward a Synthesis, 6 J.L. ECON. \& ORG. 167 (1990).

$125 \mathrm{Id}$. at 168 .

$126 \mathrm{Id}$. at 169 .

127 Id. at $168-69$. 
tend to copy other states, and the youth sports TBI lawmaking process was no exception. Perhaps the uniformity and non-invasiveness of these laws is a reflection of consistent public opinion across states or a larger cultural norm in favor of more limited interventions into matters affecting sports and youth. In addition, fiscal concerns may have prevented a more aggressive policy intervention framework in most states. Or perhaps the NFL's "feel good" message provided motivation for legislators to align themselves with a lownegative, high-positive issue to add to their campaign portfolios. Any combination of these explanations could be true, and further research is needed to focus on them individually or collectively. For this Article's purposes, the policy uniformity and the restricted range of intervention are hypothesized to be the result of interest-group engagement and messaging about the problem. To that end, the evidence gathered here (and detailed in the Appendices) focuses on the role of a key interest group, the NFL, which had the largest ability to control the shaping of the message through media, legislative engagement, and public outreach.

One sign of the NFL's influence on the policy prescriptions of state youth sports TBI laws is the high prevalence of states that included the three tenets of model legislation in their youth sports TBI laws without diverging from the NFL's suggested form of intervention. In addition to the letters of support that NFL Commissioner Roger Goodell sent to state governors urging them to pass youth sports TBI laws similar to Washington's Zackery Lystedt Law, ${ }^{128}$ the NFL also testified to legislative bodies in support of concussion safety measures. ${ }^{129}$ Furthermore, in 2011, the NCAA announced that it would join the NFL's effort to encourage state legislatures to adopt legislation similar to the Lystedt Law. ${ }^{130}$ Through these efforts, seven states passed some form of youth concussion legislation in 2010, twenty-seven states in 2011, nine states in 2012, and the remainder (absent Mississippi) in 2013. ${ }^{131}$

The evidence of the NFL's influence can best be seen by evaluating two broad metrics for determining the coupling between an interest group and the passage of laws aligned with the interest group's goals. To initially test for this influence, this Article notes the coupling of the interest group's name in news

128 See The Zackery Lystedt Law, supra note 72.

129 See, e.g., NFL Urging States to Pass Youth Concussion Laws, CBSSPORTs.COM, Feb. 23, 2011, http://www.cbssports.com/nfl/story/14729129/nfl-urging-states-to-pass-youth-concussionlaws ("NFL lobbyist Kenneth Edmonds urged DC council members to adopt rigorous concussion safety measures."). Details about the scope of testimony and other engagement can be found in the Appendix.

130 Michael David Smith, NCAA Joins NFL's Push for Youth Concussion Legislation, NBC SPORTS: PRO FoOTBALl TALK, (June 7, 2011, 4:22 PM), http://profootballtalk.nbesports.com/201 1/06/07/ncaa-joins-nfls-push-for-youth-concussionlegislation/.

131 See infra Appendix 1. 
accounts of the targeted legislation and/or its passage. In addition, the Article gathers data from a search of legislative history and/or public records surrounding the debate and eventual adoption of the state's youth sports TBI laws. In short, this initial examination of data can provide some assessment of the role of interest group influence in two critical realms-through news coverage of the youth sports TBI laws and through the legislative history of the enacted laws. Research in both realms conducted for this Article (and described in the Appendices) tracked and rated the strength of references to the NFL that appeared in conjunction with discussion of youth sports TBI laws. ${ }^{132}$ These two sets of indicators were compared to each other and analyzed according to the officially expressed support of the NFL for certain of the proposed laws. ${ }^{133}$

As detailed in the Appendices, a high-strength news reference is defined by a direct connection between the state's youth sports TBI legislative history and the NFL's official lobbying, express support, recommendations, or sponsorship, as analyzed by the reporting news source. ${ }^{134}$ High evidence of influence in the legislative history was established by one or more documents on the states' legislative websites with a direct connection between the state's legislative efforts and the NFL's official lobbying, express support, recommendations, or sponsorship. ${ }^{135}$ In the context of legislative history, the term "direct connection" denotes that materials found in the legislative record were either provided or explicitly endorsed by the NFL and thereby reasonably could be regarded as evidence of the organization's lobbying for the bill.

References, direct and indirect, to the NFL were most common in the states with laws that received its express support. ${ }^{136}$ The NFL was discussed in news accounts alongside the proposed youth sports TBI legislation in almost $75 \%$ of the states with such pending legislation. ${ }^{137}$ All of the states with strong legislative history references to the NFL received its express support except for Washington State, where the Zackery Lystedt Law functioned as the foundation for subsequent legislation. ${ }^{138}$ Even in states where the NFL did not expressly support the laws (as indicated in its public commentary, letters, and news references), the organization appears in the news and the legislative history. ${ }^{139}$ The NFL is mentioned as a driving force for states' adoption of youth sports laws regardless

132 See infra Appendices 1-2.

133 See Concussion Legislation, supra note 77.

134 See infra Appendix 1.

135 See infra Appendix 2.

136 See infra Appendices 1-3.

137 See infra Appendix 1.

138 Information about specific references to the NFL in each state's legislative history is on file with the author and available upon request.

139 See infra Appendices 1-2. 
of official influence in the development. ${ }^{140}$

Clearly, the NFL made itself a visible lobbying force throughout states' legislative processes in developing and eventually passing youth sports TBI laws. ${ }^{141}$ The question is whether the organization's motivation in doing so diverged from the public interest. The issue of youth sports concussions is directly relevant to the NFL's private commercial goals in protecting the image and reputation of football. By lobbying and providing resources and recommendations for state legislatures, the NFL was able to frame the issue (reducing negative outcomes of concussions) and shape the response accordingly (facilitating overwhelmingly uniform laws and not directly regulating the content, rules, or procedures of football itself). One plausible reason for avoiding a direct focus on the frequency or severity of the concussions themselves might have been a large class-action lawsuit against the NFL that was pending throughout much of this period. ${ }^{142}$ That lawsuit focused on the dangers of both initial concussions and repeat concussions, while suggesting that the NFL's leadership failed to put measures in place designed to minimize the risk of initial and secondary concussions.

By their submission to the NFL's influence, the states failed to carefully calibrate policy for vulnerable constituents. Where the states should act either as careful fact finders or as labs for policy experimentation, the NFL's experience dealing with football concussions and the science surrounding incidences of TBIs in sports instead served as a form of reassurance to lawmakers who trusted the NFL's judgment about the connection between policy prescription and potential policy outcomes. Although some law may well be better than none, the states' actions in failing to broaden the scope of data upon which to legislate allowed the window for optimal experimentation and policy innovation to lapse. ${ }^{143}$

Some suggest that the mere involvement of the NFL in meeting with state legislators, testifying in public hearings, and other such matters should not lead to a conclusion that its presence (direct and indirect) actually influenced legislative choices or policy outcomes. But there are two possible reasons why this is likely untrue. First, by defining the problem as "re-injury following re-entry", the NFL and its focus on the Lystedt story directed attention away from a different public health problem-the initial injury. Thus, focusing legislators on solving for the problem of injury after re-entry necessarily led away from more holistic policy

140 See infra Appendix 1.

141 See infra Appendix 1.

142 See, e.g., Andrew Brandt, The Other Lawsuit, MMQB with Peter KING (Nov. 9, 2013), $\mathrm{http}: / / \mathrm{mmqb}$.si.com/2013/10/25/riddell-lawsuit-nfl-concussions-andrew-brandt/.

143 The NFL is one of many important advocacy groups working to raise awareness and seek legislative resolution for the issues of youth concussions. The legislative influence of the NFL may also be consistent with the supporting efforts of many other organizations that also appear as advocates throughout the news stories and the states' legislative histories. 
prescriptions designed to focus on initial prevention. Second, policymakers must be influenced by something, whether it is moral values, public opinion, culture, religion, or interest groups. And in the context of public health, direct personal contact with researchers and health advocates serves as the most important driver of policymaking choices in a given public health intervention. ${ }^{144}$ Further, policy makers in public health settings tend to rely on sources that they trust and for whom they have access. ${ }^{145}$ The NFL provided both access and a trustworthy message given its potential interest in reducing the overall incidence of concussions in sports generally, or at least football in particular. Thus, it seems more likely than not that the NFL's message and influencing served as one key motivation for the adoption of youth sports TBI laws.

The second motivation for the widespread adoption of youth sports TBI laws is the increased public understanding and awareness of the frequency of youth concussions. In addition to the concussion awareness campaign being led by the $\mathrm{NFL},{ }^{146}$ other organizations have taken steps to raise awareness. For example, the National Academy of Neuropsychology and the National Athletic Trainers' Association partnered on a campaign to raise awareness on concussions and concussion treatment. ${ }^{147}$ As a result of these efforts, more people-and certainly state legislators-became aware in recent years of the dangers that a concussion poses to a young athlete. Youth TBI laws protect children and raise awareness of an issue of public importance with few public opponents-factors that would typically reflect positively on a legislator's public persona and electoral positioning.

The third factor influencing the content and adoption of youth sports TBI laws is likely a desire to minimize state budget expenditures while providing a policy solution to a costly problem. This survey of youth sports TBI laws shows that the vast majority of states do not require new programs or costs to either state agencies or individual school districts. ${ }^{148}$ Rather, the concussion policies are

144 See, e.g., Simon Innvaer et al., Health Policy-Makers' Perceptions of Their Use of Evidence: A Systematic Review, 7 J. Health Services Res. \& Pol'y 239, 239-44 (2002) (reviewing studies and interviews with health policymakers and identifying personal contact as a key policy-choice making variable).

145 See, e.g., Christopher A. Jewell \& Lisa A. Bero, Developing Good Taste in Evidence: Facilitators of and Hindrances to Evidence-Informed Health Policymaking in State Government, 86 MiLbank Q. 177 (2008).

146 See generally Roger Goodell Q\&A: "We Can Make the Game Safer", NFLEvOLUTION.COM (Sept. 5, 2012), http://www.nflevolution.com/article/Roger-Goodell-Q-038A-8216-We-can-make-the-game-safer-8217-?ref $=1299$.

147 National Academy of Neuropsychology (NAN) and National Athletic Trainers' Association (NATA) Team up on Campaign to Raise Concussion Awareness, NHL.COM (Sept. 9, 2009, 5:03 PM), http://www.nhl.com/ice/news.htm?id=499020.

148 See, e.g., Colo. Legis. Council StafF, State and Local Revised Fiscal Impact, S.B. 11-040, (Colo. 2011), available at http://www.leg.state.co.us/clics/clics2011 a/csl.nsf/fsbillcont3/ 
expected to increase a state agency or school's workload but not require additional funding. ${ }^{149}$ In fact, the NFL on its Frequently Asked Questions about Concussion Prevention Laws webpage ${ }^{150}$ explains that the cost of implementing a concussion prevention and awareness bill similar to the Zackery Lystedt Law is zero since the three tenets contain "no requirements that resources be spent to hire or train medical professionals or to purchase equipment." general information on effective concussion management practices is publicly available and free through the Centers for Disease Control for high school and youth coaches, parents, athletes, and school professionals. ${ }^{152}$

Through the combination of these three motivating factors, all of which relate to the NFL's influence, states rapidly adopted largely identical youth sports TBI laws. While enactment of a youth sports TBI law modeled on the Lystedt law is generally a positive step toward reducing the number of traumatic brain reinjuries among young athletes, these laws could still be improved in a variety of ways-particularly by focusing on primary prevention. It is troubling that states only began to act rapidly on this issue by adopting NFL-endorsed policies from a consistent one-size-fits-all model once the NFL's organizational lobbying began. This kind of interest-based TBI-prevention policy choice framing could result in solutions that primarily reflect the interest groups' concerns rather than the public interest overall. In addition to the legislative innovations above, there are a variety of other ways that state legislative interventions could more effectively address the problem of youth concussions. Section IV now turns to those interventions.

\section{FaIluRes of EXISTing Policy ANd Potential PoliCy IMPRovemEnts}

The aforementioned interventionist public health law approaches to concussions have many common features, a few innovations, and a high degree of uniformity. All but a few suffer from three critical shortcomings. First, they fail to include evaluative metrics to determine whether the law's reforms are helping to solve the problem. Second, existing youth sports TBI laws have a singular focus on reducing the secondary efforts of concussions rather than attacking their root causes. Finally, existing youth sports TBI laws fail to track individual athletes and the rise in risk associated with athletes who suffer multiple concussions. These factors may undermine the efficacy of existing

A9CE9CEE 12645CAA8725780800800D80?Open\&file=SB040_rl.pdf. 149 Id.

150 Frequently Asked Questions About Concussion Prevention Laws, NFLHEALTHANDSAFETY.COM, http://nfl-www.fkhstaging.com/map/FAQ.php (last visited Dec. 5, 2013).

151 Id.

152 Id. 
youth sports TBI laws; solutions are proposed here to remedy that effectiveness gap and potentially address root causes. These are just some of an array of potential policy innovations, but their efficacy can only fully be valued by policymakers with a more robust and consistent research agenda and use of data-which at the moment all but a few states do not collect or analyze for trends. ${ }^{153}$

\section{A. Mandatory Aggregate Reporting and Feedback Regimes}

The most perplexing omission in youth sports TBI laws is the failure of almost all states to develop a reporting and testing system to evaluate the effectiveness of the laws. One would think that testing, evaluation, and science should be at the core of any broad-based public health initiative. But only three states have (indirectly) mandated a surveillance program by implementing regulation or mandatory general reports. ${ }^{154}$ These provisions or regulations will allow forward-thinking states to test and report on the effectiveness of their newly created youth concussion regimes, while others will not have a systematic method of evaluating the law's impact. While this would entail a cost to the entity charged with producing the report, an analysis of the youth concussion data is the primary way to determine whether the law is actually effective. Since states have recently reduced their general expenditures drastically, ${ }^{155}$ state legislators may be relying on the press to investigate the effectiveness of the youth concussion measures in lieu of a state study, though there is not specific evidence to support this contention. ${ }^{156}$ Given that one purpose of state-based concussion lawmaking could have been to help reduce both the incidence and long-term health consequences of concussions, this failure to include an evaluative metric will reduce the amount of information available to

153 See, e.g., Abby S. Haynes et al., Galvanizers, Guides, Champions, and Shields: The Many Ways that Policymakers Use Public Health Researchers, 89 MiLbank Q. 564 (2011).

154 See, e.g., Mo. REv. STAT. § 167.775 (2013) ("Any statewide athletic organization with a public school district as a member shall be required to publish an annual report relating to the impact of concussions and head injuries on student athletes which details efforts that may be made to minimize damages from injuries sustained by students participating in school sports.").

155 See Fiscal Survey of States: Spring 2011, NAT'L GoverNorS Ass'N \& NAT'L ASS'N OF State BUDGET OfFiCERS, at viii (2011), http://www.nasbo.org/sites/default/files/Spring-2011Fiscal-Survey_0.pdf ("Twenty-three states made budget cuts to their fiscal 2011 budgets totaling $\$ 7.8$ billion. Thirty-nine states made mid-year budget cuts of $\$ 18.3$ billion in fiscal 2010 , while 43 states made $\$ 31.3$ billion in mid-year cuts in fiscal 2009.").

156 However, an innovative grant-funded program was recently developed to begin making such an assessment of effectiveness. See Evaluating Implementation of Return-to-Play Laws for Athletes with Concussions to Increase Effectiveness of Existing and Future Legislation, ROBERT WOOD JOHNSON FOUND., http://www.rwjf.org/en/grants/grant-records/2011/11/evaluatingimplementation-of-return-to-play-laws-for-athletes-wi.html (last visited Dec. 5, 2013). 
policymakers to determine whether and how the law is helping to reduce the incidence of primary and secondary TBIs. ${ }^{157}$

\section{B. Mandatory Individual Tracking Metrics}

Existing youth sports TBI legal regimes fail to include player-level tracking systems designed to ensure that a youth athlete's medical history is tightly tied to treatment and outcomes. ${ }^{158}$ Because the epidemiology of TBIs is relatively consistent with respect to the increased level of risk for athletes who have suffered repeated concussions, states have a strong interest in monitoring the overall number of concussions sustained by athletes. First, with respect to shortterm health, medical professionals generally agree that the recovery time for concussions increases when one has suffered a previous concussion. Second, as described earlier, CTE and other long-term health outcomes appear to be tied to a history of repeated concussions. Only by tracking the concussion history for each student athlete can a state's public health officials, school districts, coaches, and parents be certain that the medical response to an athlete's concussion is appropriately tied to the athlete's previous history. The CDC agrees, identifying "collecting data from schools" and "studying changes in concussion knowledge ... before and after the policy is put in place." 159 Vermont and Massachusetts are pioneers in this effort, but other states have not taken this initiative. It is true that such efforts could be costly, but given the already required intervention for return-to-play clearance, it hardly seems more onerous to notate a school's master file or record about the occurrence. But assuming that a state's true concern is cost, others have suggested that the CDC develop and implement a national surveillance system, thus potentially removing cost as a barrier. ${ }^{160}$

\section{Required Baselines}

Professional leagues and some states also rely on pre/post-concussion evaluative baseline player metrics to determine when a player's cognitive

157 The failure to include such information in reporting could be explained as a cost-saving mechanism. In addition, the visibility of such data could complicate policy adherence and overall enforcement, since individual schools or coaches might face additional scrutiny if such rates were substantially higher than the state's average.

158 However, Massachusetts and Michigan youth sports TBI laws suggest that such a function is possible under their regimes. MASS. GEN. LAws ch. 111, § 222(b) (2013); Мich. COMP. LAWS $\S \S$ $333.9155,333.9156(2013)$.

159 See Nat'l Ctr. for Injury Prevention \& Control, Get a Heads Up on Concussion in Sports Policies: Information for Parents, Coaches, and School \& Sports Policies: Information for Parents, Coaches, and School \& Sports Professionals, CDC, http://www.cdc.gov/concussion/pdf/HeadsUpOnConcussionInSportsPolicies-a.pdf (last visited Dec. 5,2013$)$.

160 See, e.g., Sports-Related Concussions, supra note 47. 
functions substantially deviate from a pre-established "norm"; players demonstrating such deviations would not be permitted to reenter athletic competition until their cognitive functioning returned to normal. In professional sports, baseline tests are the norm for players. ${ }^{161}$ Across youth sports, such tests are not yet common practice. The evaluation of TBIs at the professional level is useful as a guiding lesson for high school sports programs but may also be a financially unrealistic model for states absent partnerships with universities, donors, and others who provide the baseline testing vehicles. ${ }^{162}$ Yet some states such as New Jersey and Rhode Island have still managed to develop regimes that build in baseline testing. However, as discussed earlier, the effectiveness of baseline testing is not empirically certain.

\section{Direct Intervention in Particular Sports}

All but one or two provisions across the entire set of state youth sports TBI laws are targeted to reducing secondary TBI risks-those short- and long-term health consequences that begin after an athlete has already suffered a concussion. Reading pamphlets about how to identify when players have a concussion, making them sit out when a concussion is suspected, and having them evaluated by a doctor are all well-timed and important state-law reforms, but they all take the existence of a concussion for granted.

Why this near-exclusive focus on what happens after a TBI has already occurred? Part of the failure to address the underlying issue itself - prevention of initial TBIs - is that to do more would require a more invasive legislative and financial mandate than some states may be willing to implement. ${ }^{163}$ An additional factor is rooted in the nature of competition itself. The sports that are most likely to cause concussions (cheerleading, football, hockey, and soccer) are all popular team sports cutting across regional, gender, and cultural lines. Therefore, while it is obvious that not offering these sports reduces the likelihood of concussions within a given youth population, such an option might be politically and culturally untenable. $^{164}$ The focus of TBI laws on results of concussions, instead

161 See, e.g., Standardized Concussion Tests Coming to the NFL, NFL, http://www.nfl.com/news/story/09000d5d81 e76bed/article/standardized-concussion-tests-comingto-the-nfl (last updated July 26, 2012).

162 New Jersey's approach to baseline testing is unique. It applies a small surcharge to carregistration fees, which is then used, in part, to pay for baseline neuropsychological testing of highschool student athletes. See N.J. STAT. ANN. § 18A:40-41.7 (West 2013).

163 Because states were so focused on the NFL-endorsed three tenets, scant evidence exists that any substantial alternatives to the Lystedt model were considered. That having been said, a few states, discussed herein, did engage in some policy innovation.

164 This being said, President Theodore Roosevelt once vigorously and successfully campaigned for more rigorous safety standards in football, spurring substantive game-changing rules (like the invention of the "forward pass"), as well as the creation of national sport oversight 
of reducing concussions to begin with, may also have to do with the social value of youth sports activities and desire to avoid directly changing the customary rules of a game. ${ }^{165}$ That youth sports TBI laws primarily focus on what happens after a TBI presupposes the legitimacy of the event that caused the TBI. That sports, and particularly football, have nationally recognized social (and perhaps even political) import and value speaks again to why a powerful private interest group like the NFL would seek to influence the debate and shape the solution from the beginning.

Aside from the extreme position of eliminating popular sports, there are still other direct sport interventions that likely would reduce the overall number of concussive events, including the mandatory improvement of equipment and changes in style of play or specific in-sport maneuvers. ${ }^{166}$ Yet while the exact science of how helmets reduce or minimize risk of concussions is still uncertain, it is probable that newer helmets will help eliminate that risk more effectively than older ones. ${ }^{167}$ A legislative mandate that organized football programs buy entirely new helmets every two to three years may reduce the number of concussions in youth football, but requiring new helmets so often would also substantially drive up the costs to schools and school districts. ${ }^{168}$ Because helmets are an expensive part of the game, teams have incentives to minimize their constant replacement. However, because helmets can be partially remanufactured for a much lower cost than a new helmet (both new materials

regimes like the NCAA. Jim Morrison, The Early History of Football's Forward Pass, SMITHSONIAN.COM (Dec. 28, 2010), http://www.smithsonianmag.com/history-archaeology/TheEarly-History-of-Footballs-Forward-Pass.html.

165 For example, researchers recently used advanced MRI techniques to show that frequent heading of the ball in soccer resulted in TBI-like symptoms. See, e.g., Frequent Soccer Ball "Heading" May Lead to Brain Injury, Albert Einstein Coll. Med. OF Yeshiva Univ. (June 11, 2013), http://www.einstein.yu.edu/news/releases/915/frequent-soccer-ball-heading-may-lead-tobrain-injury/. However, because heading the ball is a core component of soccer play enjoyed by fans and players alike, it is difficult to imagine a law-based regime aimed at changing that sport's core play not suffering from public resistance.

166 The NFL and other sports leagues have, in recent years, focused on penalizing certain head-impacting maneuvers, such as when the NFL began penalizing players in 2013 who engaged in running or tackling by leading with the crown of their helmets. See, e.g., Ian Rapoport, Rule Change Banning Head-On Approach Comes with Questions, NFL, http://www.nfl.com/news/story/0ap 1000000151942/article/rule-change-banning-headon-approachcomes-with-questions (last updated Mar. 20, 2013).

167 See, e.g., Jenny Vrentas, The First Line of Defense, MMQB with PeTER KING (Oct. 22, 2013), http://mmqb.si.com/2013/10/22/nfl-helmets-head-injury-concussion/ (describing safety features implemented in newer helmets and the current competitive market for helmet innovation and safety).

168 Although there is not a direct correlation between age of helmet and TBI prevention, once helmets reach a certain age, effectiveness as even a baseline head injury protection decreases. See, e.g., Schwarz, supra note 63 (examining helmet standards in youth sports and noting that "[m]ore than 100,000 children are wearing helmets too old to provide adequate protection"). 
and/or new design), older helmet designs can remain in circulation for far longer than some experts believe is useful. ${ }^{169}$

A full discussion of the "helmet debate" is beyond the scope of this Article. Nonetheless, a growing body of literature suggests the lack of long-term evidence regarding the quality and effectiveness of football helmets in preventing TBIs. ${ }^{170}$ As those studies explain, football helmet standards have not changed meaningfully since $1973,{ }^{171}$ and the studies undertaken to test their effectiveness do not approximate real world conditions. Further, helmet standards are regulated by a private consortium and are not subject to rigorous reporting requirements. Therefore, it is not certain that existing helmets are particularly safe. In this highly uncertain environment, when one considers that mandatory helmet replacement may also be particularly difficult for low-income parents and athletes, one can see how the status quo is maintained.

Finally, with respect to sports-based interventions, youth sports TBI laws have generally taken a one-size-fits-all approach. They do not focus on how changes in play in individual sports might reduce the primary instances of youth sports TBIs. There may be many reasons for this, ${ }^{172}$ but the laws do not acknowledge the scientific consensus that youth TBIs vary on the basis of age, the type of sport, and whether the athlete is male or female. ${ }^{173}$ In the future, there may be value in legislation that addresses organized sports risks in a more specific manner. For example, because a significant proportion of TBIs suffered by female youth student-athletes occur in soccer and cheerleading, more narrowly or finely tailored laws might direct state officials to involve soccer or cheerleading sport-specific associations in the development of TBI identification and treatment guidelines. Or, because most male youth student-athlete TBIs occur in soccer and football, TBI laws may maximize their impact by heightening sport-specific TBI reduction techniques. It is also possible that race and socioeconomic factors play a role, resulting in a race- or class-skewed level of TBI incidences across sports. However, lacking data beyond anecdotes, it is

169 For example, in an incident described by Lazarus, an NFL player's helmet design was originally developed in 1988, but was being worn many years later when an incident occurred, despite subsequent improvements to helmet design. See Arthur Lazarus, NFL Concussions and Common Sense: A Recipe for Medical Errors and a Lesson for Physician Leaders, 37 PHYSICIAN EXECUTIVE J. 6 (2011) (describing an NFL game where two players with on-field concussions were removed and then re-inserted in the same game, despite both of them having typical concussive indicators).

170 McCrory et al., supra note 94 , at 255 (suggesting no proven link to preventing TBIs).

171 See, e.g., Alan Schwarz, Soon, Helmet Data at a Keystroke, N.Y. Times, Jan. 9, 2011, $\mathrm{http}: / / q u e r y . n y t i m e s . c o m / g s t / f u l l p a g e . h t m l$ res=9805E2D9143DF93AA35752C0A9679D8B63.

172 See Jewell \&Bero, supra note 145.

173 Andrew E. Lincoln, Trends in Concussion Incidence in High School Sports: A Prospective 11-Year Study, 39 AM. J. SPORTS MED. 958 (2011). 
difficult to determine whether this is broadly true.

Separately, legislative mandates about how the games must be played (for example, eliminating certain types of hits or maneuvers, or reducing the amount and/or intensity of practice drills for certain sports) would also certainly reduce risk and drive overall concussion rates downward. The CDC agrees, identifying "rule changes" and banning or limiting "certain drills or techniques" in sports in an attempt to reduce injury. ${ }^{174}$ However, such mandates would directly interfere with the inner workings of a sport and would be vigorously challenged by those who play such sports. These three direct intervention alternatives (eliminating certain sports, imposing additional equipment expenses, changing rules) can each reduce the overall number of concussions, but not without potential public backlash or limitations due to fiscal constraints across districts and states. Therefore, it has been easier for lawmakers to focus on secondary interventions, where the core nature of the sports themselves is not directly threatened. Evidence presented here should lead states and districts to experiment with an array of approaches, which would allow policymakers to gather evidence to determine which interventions, changes, and regulations are the most effective at reducing incidences both initial and repeat youth sports TBIs.

\section{E. Fixing the Reporting Disincentive}

Most analysts agree that the incentives to clear concussed players to return to play places teams, doctors, and athletes in asymmetrical positions. ${ }^{175}$ Sound longterm medical opinions cannot always be provided in a high-pressure situation where quick decisions are needed. As such, the more that state-level reforms can successfully disentangle these incentives, the more successful these reforms are likely to be in reducing short-term re-injury and longer-term consequences. However, when a physician or health professional has a management position with a team, the tension between protecting patients (players) and maximizing team value (winning the game) seems like an obvious conflict that should be avoided. Certainly, coaches face similar incentives to potentially prioritize winning over player safety. ${ }^{176}$ As noted above, two states have attempted to address this concern by creating punitive enforcement mechanisms for failing to report TBIs. Another way might be to create an anonymous reporting structure, modeled after whistleblower provisions in other laws. The provision could allow parents, athletes, and other officials to share information when they have reason to believe that the state's TBI mandates are not being followed.

174 See Nat'l Ctr. for Injury Prevention \& Control, supra note 159.

175 See, e.g., Michael McCrea et al., Unreported Concussion in High School Football Players: Implications for Prevention, 14 CLINICAL J. SPORT MED. 13 (2004).

176 See, e.g., Brendan L. Smith, Benched Coaches Shooting Back, A.B.A. J. (Oct. 1, 2010, 2:00 AM), http://www.abajournal.com/magazine/article/benched_coaches_shooting_back/. 


\section{F. Learning from Professional Sports and Independent Associations}

There is no shortage of conclusions, speculations, and recommendations about how to assess the TBI problem, minimize its effects, and prevent injuries in both adults and children. We now know that concussions occur in youth sports more frequently when inexperienced athletes are playing in team sports. ${ }^{177} \mathrm{We}$ also know that ice hockey and football participants are the most likely to suffer from concussive events. ${ }^{178}$ And we know that professional sports organizations have undertaken tough new measures designed to minimize the risk and consequences of initial concussions, and also to prevent multiple TBIs. These measures include changes in equipment, sports rules, or the times and locations in which sports are played. ${ }^{179}$ The lessons of these varied approaches can be instructive.

Both the NFL ${ }^{180}$ and the National Hockey League (NHL) have changed rules with the explicit intent of reducing TBIs. ${ }^{181}$ At the collegiate level, the NCAA has partnered with the CDC to promote concussion safety and best practices. ${ }^{182}$ Major League Baseball's aggressive concussion protocols require: (1) baseline testing for all players and umpires, (2) strict evaluation of individuals who have suffered concussions, and (3) the creation of a seven-day disabled list for players with concussions. ${ }^{183}$ Independent associations also have stricter post-concussive guidelines than are implemented by state laws. For example, the American Academy of Pediatrics (AAP) cautions that a youth athlete who has suffered a concussion should be gradually reintroduced into athletic activities over a fiveday period to ensure that there are no residual signs of concussive effects. ${ }^{184}$ In youth sports organizational bodies, similar efforts are underway to establish clear guidelines and recommendations for various sports. ${ }^{185}$ Most recently, Pop Warner

177 Mitka, supra note 18 , at $1775-76$.

1782009 Participation - Alphabetically, NAT'L SPORTING GOODS Ass'N, http://www.nsga.org/files/public/2009_Participation-Alphabetically_4Web_100521.pdf (last visited Dec. 6, 2013).

179 Christine Provvidenza \& Charles H. Tator, Sports Injury Prevention: General Principles, in Catastrophic Injuries in Sports and Recreation: Causes and Prevention-A Canadian STUDY 59 (Charles H. Tator ed., 2008).

180 See Memo Explains Policy to Have Trainers Monitor for Concussions, NFL, http://www.nfl.com/news/story/09000d5d82547e65/article/memo-explains-policy-to-have-trainersmonitor-for-concussions (last updated July 26, 2012).

181 See Lazarus, supra note 169.

182 Attention College Sports Fans: CDC and NCAA Team Up on Concussion Safety, CDC, http://www.cdc.gov/concussion/sports/cdc_ncaa.html (last updated Mar. 9, 2012).

183 See Erick Almonte Slept Under Supervision, ESPN, http://sports.espn.go.com/mlb/ news/story?id=6437072 (last updated Apr. 27, 2011).

184 See Mark E. Halstead, Sport-Related Concussion in Children and Adolescents, 126 Pediatrics 597, 604-05 (2010).

185 See, e.g., AACCA Concussion Management and Return to Play Protocol, AM. Ass'N OF 
(an umbrella organization for youth football, cheer, and dance programs in fortytwo states) created new standards to limit contact during football practice and heighten concussion awareness among participants. ${ }^{186}$

In comparison to the approaches adopted by professional sports and other national associations, states have adopted minimalist strategies, particularly with respect to return-to-play provisions. Recall that the science is fairly clear even in its ambiguity: there is simply no strong evidence to determine when an athlete is well enough to return to competition or which intervention method following a TBI works best to speed recovery. ${ }^{187}$ And while the MLB and AAP's timetables are considered scientifically reasonable, the scientific evidence about the scope and length of post-concussive re-entry is still disputed. ${ }^{188}$ At a minimum, one might suggest that the graduated assessment regimes of professional sports organizations should be a floor, not a ceiling, for state law innovations involving vulnerable athletes. Yet no state law sets out a graduated timetable for return to play or evaluation, instead relying on the now-standard minimum 24-hour waiting period. This is not to say that relying on professional sports standards is a policy panacea. For example, return-to-play decisions in the NFL are not uniformly followed and could actually serve as negative guidance or modeling for youth programs. ${ }^{189}$ Nonetheless, the policies created by professional and independent associations should not be entirely ignored by states.

\section{G. Evaluating Policy Alternatives}

Each of the alternative policy reforms described above addresses substantive lawmaking choices in existing youth sports TBI laws, suggesting that more can be done by way of innovating with TBI reduction measures in youth sports TBI laws. ${ }^{190}$ Based on the evidence presented here, a truly comprehensive youth sports TBI prevention framework should reduce the overall number of youth head injuries, prevent secondary head injuries, and potentially reduce the (as yet unknown) long-term effects of head injuries caused by athletic competition. But

\footnotetext{
CheErleading COACHES \& Administrators, http:/www.aacca.org/content .aspx?item=/Resources/concussions.xml (last visited Dec. 5, 2013).

186 See Pop Warner Leagues Limit Contact in Practice, SI.COM (June 12, 2012, 8:41 PM), http://sportsillustrated.cnn.com/2012/more/06/12/pop.warner.contact.ap/index.html.

187 Mitka, supra note 18 , at 1775-76.

188 Recall that one expert testified that brain-injury proteins linger up to three months after initial injury. See notes 64-66 and accompanying text.

189 See Lazarus, supra note 169.

190 This discussion focuses only on reduction of short-term health consequences and non-fatal TBI-related events. Morbidity and mortality are commonly associated with all types of TBIs in children, yet reforms in youth sports TBI laws do not directly address the study of morbidity and mortality with respect to youth TBIs developed outside of athletics and compare interventions for those TBIs with ones that might be appropriate in youth sports. See, e.g., Carol A. Hawley, Behaviour and School Performance After Brain Injury, 18 BRAIN INJURY 645, 645-49 (2004).
} 
the real issue is the overall scope of reduction in youth TBIs, not whether some negligible rate reduction can be achieved through new state laws. Nonetheless, there are those who argue that alternative frameworks- - or no frameworks at all-would be better than the laws studied here.

Prior to the enactment of the Zackery Lystedt Law in Washington State, public health interventions and interest group efforts, while well-meaning, did not appear to have a measurable effect on the overall volume of concussions in youth populations. ${ }^{191}$ During the era preceding the Zackery Lystedt Law, the CDC set forth vigorous, clear, and helpful guidelines that were available to coaches, youth athletes, and their families. Yet without the drive, knowledge, and enforcement power of state law to ensure that this information reached those populations, there is little evidence that any of the information had a substantive effect on the goal of reducing TBIs in youth populations.

Such motivation, information, and power have recently been provided, in part by the NFL, and seem to have finally encouraged the states to act. In this post-Zackery Lystedt Law era of public health law reform, the issue has been dominated by the NFL's effective interest group advocacy. The regulatory capture of youth sports TBI lawmaking by such a powerful interest group is one key reason why the underlying policy discussion has failed to exhibit any real experimentation, and why the resulting policies lack the innovative spark that states' independent legislative debates could have provided.

\section{CONCLUSION}

We know that youth TBIs and their residual effects are a serious problem, with a complex web of causal factors and ensuing consequences that remain poorly understood. Legislative mandates have been enacted in all but a single state with the express purpose of providing information and establishing minimum responses to TBI events, presumably with the laudable goal of reducing the overall number of youth TBIs, minimizing their residual and repeated impact, and providing for safer outcomes in youth sports. All of these goals are worthy of public praise, and some state action was surely better than state silence. All agree that youth sports place youth at risk of suffering TBIs, yet public health laws fail to foster assessment and reporting mechanisms to evaluate and minimize these well-known risks and health outcomes. The Zackary Lystedt Law and the three tenets it helped to define provided a useful framework to begin analyzing how the law might help address youth sports TBIs, but it does not have to be the end goal.

As the above analysis shows, the three tenets are simply not enough to

191 It is possible that, despite the documented rise in reported TBIs, such efforts did reduce the pace of that rise. Unfortunately, there are no accurate measures to test this hypothesis. 
accomplish the long-term youth TBI reduction goals that states could be (and arguably should be) attempting to reach. It may be that heightened attention to these secondary prevention measures could reach back to some initial prevention solutions, but without knowing precisely what those initial prevention measures are, it remains unclear how state law could successfully reach toward them. Laws that require a mandatory sitting-out period, awareness by coaches, parents, and players of concussion signs, and medical evaluation of concussed players all represent worthy initiatives that focus on helping recognize when an athlete is already in a concussive or post-concussive state. These initiatives do not appear on their face to address TBIs in the first instance-i.e., the reduction of the risk factors that might cause the initial concussion itself. Because legislative focus on the Lystedt Law's three tenets has achieved relatively uniform post-concussive initiatives, it may be time to consider whether this unique state/private-actor partnership should be expanded to include additional interest groups, broader metrics, and additional and more comprehensive proposals for reform. It seems clear that the NFL's influential role at least partly explains why more innovation has not flourished, despite the differences in politics, culture, and sports across the 49 states and the District of Columbia. ${ }^{192}$ This is not to say that these laws accomplished nothing, but to suggest that the definition of the problem, helped along by the NFL, has not led to substantive discussion about policy alternatives or a broader framing or focus on youth sports TBI's more generally.

Further study of youth sports TBI laws over time is needed to test their efficacy and both secondary and primary risk reduction. But to do so most effectively, either more policy innovation or more evaluative metrics are required at the state level. Ideally, research would take a state-by-state survey of the field, examine variation in various state youth sports TBI laws, and somehow evaluate the successful or innovative traits across such laws by comparing longitudinal policy outcomes in the populations targeted by these laws. However, as has been explained, existing laws exhibit very little variance across states. Therefore, such research might take the form of case studies, whereby researchers would select a few states with radically different youth sports TBI laws, with another as a control, and research how states with stronger, mid-range, weaker, or no laws protected or enhanced public health outcomes with respect to youth sports TBIs. Additional research could take a quantitative approach, drawing upon data trends across states (such as rates of concussions pre/post-enactment) and could determine whether the regimes have had some effect.

Unfortunately, comprehensive evaluative and innovative public health law

192 In other contexts, this phenomenon could be considered a form of groupthink, causing policy paralysis in state youth TBI lawmaking as a result of a lack of dissention or competing voices in the debate. See, e.g., IRVING L. JANIS, Victims OF GROUPTHINK: A PSYCHOLOGICAL STUDY of Foreign-Policy Decisions and Fiascoes (1972). 
studies of youth sports TBI laws and their outcomes are made extraordinarily difficult by the failure of states to include robust reporting mechanisms in their youth sports TBI laws. If policymakers are serious about using the force of the law to have an impact on public health, they must also create evaluative metrics to ensure that their lawmaking has the desired effect on public health outcomes. The lack of required evidence gathering for such an important policy goal is both surprising and counterintuitive. If the stated goal of such legislation is to reduce the overall number of TBIs and to minimize their long-term consequences, the only way that one can test whether a state's law is effective is by (a) creating a baseline assessment for all student athletes, (b) creating a reporting regime that requires coaches, hospitals, and schools to publicly identify instances of youth TBIs across a variety of variables (sports, school districts, etc.), and/or (c) repeating baseline assessments on student athletes over time to measure any potential decline in performance and determine whether such decline might be caused by an athlete's injury. The long-term effort to reduce youth sports TBIs must involve policy evaluation, policy experimentation, or measurement of policy outcomes in youth populations. Only when states choose to gather and assess information tied to their policies can they best determine whether the policies are helping to achieve their goals-and if not, how they might change the policies to be more effective. Even though all states but one now have such youth sports TBI laws, the policymaking game is not over. The risk-reducing steps taken in response to the public health concerns described here were just the beginning of a long season that is far from over. 


\section{APPENDICES $^{193}$ \\ Appendix 1: Evidence of Influence-News}

A LexisNexis news search was run twice, once from major newspapers and once from regional news sources across all fifty states and the District of Columbia. The search included a terms \& connectors search across the last five years for "National Football League" or NFL in the same paragraph as brain or concussion or TBI, student or youth or child, and legis! or bill or act. ${ }^{194}$

Out of the then 45 states (including the District of Columbia) with youth sports TBI laws, 33 were mentioned in combination with references to the NFL in these news sources. These references were assigned strengths of high, medium, or low.

A low-strength reference includes only a passing mention of the name of the state in a list while discussing ongoing legislative efforts across other states, albeit in combination with some mention of the NFL in the greater discussion. ${ }^{195}$ Seven states have an NFL news reference strength of low. ${ }^{196}$

A medium-strength reference includes mention of the state in a statement connecting it with the NFL but without an explicit reference to a lobbying, supporting, or otherwise sponsoring relationship. Sometimes this involves mention of a former NFL player's involvement in the legislative efforts. ${ }^{197}$ Other times this involves naming the state in a list, similar to the low-strength references, but qualifying the list of states by suggesting that they have been influenced by the NFL in an accompanying remark. ${ }^{198}$ Nine states have an NFL

193 This analysis was conducted in January and February of 2013, before several states passed youth sports TBI laws or changes to existing TBI laws.

194 ("National Football League" or NFL) /p (brain or concussion or tbi) /p (student or youth or child!) /p (legis! or bill or act) and date geq (12/11/2007)

195 For example: "Other football-laden states like Alabama[,] . . Nebraska and Oklahoma have all passed youth-concussion legislation within the past two years." Shannon J. Owens, Florida Lagging Behind on Youth-Concussion Prevention, OrLANDo SENTINEL, Aug. 1, 2011, http://articles.orlandosentinel.com/2011-08-01/sports/os-shannonowens-youth-concussions-

20110801_1_concussions-youth-sports-league-rich-football-history.

196 The seven states are Alabama, Iowa, Minnesota, Nebraska, Oklahoma, and Wyoming, as well as the District of Columbia.

197 For example: "Becker, a former offensive lineman and nine-year NFL veteran, was in Springfield [Illinois] Wednesday to promote legislation ...." Jeff Engelhardt, Former Bear Raises Concussion Awareness, CHI. DAILY Herald, Feb. 23, 2011, http://www.dailyherald.com/article/20110223/news/702239919/.

198 For example: "[L]awmakers in . . . Connecticut, Massachusetts . . . and Rhode Island legislatures are debating similar laws. ... All of these states have or are contemplating adopting some version of the National Football League rules . . . Juanita Thornton, Brain Injuries Are Serious, RoANOKE Times, Mar. 2, 2010, at A13. 
news reference strength of medium. ${ }^{199}$

A high-strength reference includes a direct connection between the state's legislative efforts and the NFL's official lobbying, express support, recommendations, or sponsorship. ${ }^{200}$ Seventeen states have an NFL news reference strength of high. ${ }^{201}$

Twelve states had no NFL news references. Although these states were sometimes referred to in published news articles, the referenced article was unrelated to the accompanying NFL term that prompted a "match" under the search protocol. Sometimes this occurred when one state's newspaper discussed other states' legislative activities in relation to the NFL. Other times this happened when an article combined two topics, discussing both youth sports TBI laws (and referencing the NFL in combination with such) as well as related traumatic brain injury resources available in other states. ${ }^{202}$

\section{Appendix 2: Evidence of Influence-Legislative history}

A legislative history search was conducted through each of the 45 state legislatures' websites. ${ }^{203}$ Although the websites are not uniform in their layout or content, the laws can all be found through a few methods. Some states' sites offer an embedded search with preset query boxes for the document type (such as bill, senate or house file, or legislative document), number, and year. Other states' sites offer clickable links for legislative history with searchable indexes organized thereunder. Still others states' sites offer only a general search query box. Most states' sites offered two or more of these search methods.

Once the enrolled law was found as a document on the legislative websites, the preceding or linked pages usually provided accompanying documents. The availability and depth of these resources varied greatly. Law-related documents included sponsor statements, statements of intent, fiscal notes, minutes and audio, committee actions, sectional analyses, suggested amendments, news stories, and academic articles, among others. Where available, these documents were

199 The nine states are Connecticut, Illinois, Massachusetts, New Mexico, Oregon, Rhode Island, South Dakota, Utah, and Virginia.

200 For example: "Adding support to the legislation at the Capitol press conference were Joe Browne, senior adviser to NFL Commissioner Roger Goodell . . . Stevenson Joins Effort to Reduce Youth Sports Injuries, PA. House Republican Caucus (Jan. 28, 2011), http://www.pahousegop.com/NewsItem.aspx?NewsID=10371.

201 The 17 states are Arkansas, Arizona, California, Colorado, Florida, Kentucky, Louisiana, Mississippi, Missouri, New Hampshire, New Jersey, New York, Ohio, Pennsylvania, Texas, Washington, and Wisconsin.

202 The twelve states are Arkansas, Delaware, Hawaii, Idaho, Indiana, Kansas, Maine, Maryland, Nevada, North Carolina, North Dakota, and Vermont.

203 As described above, see supra note 193, this analysis was conducted in early 2013, prior to several states' adopting or changing existing youth sports TBI laws. 
searched for any mention of NFL or National Football League.

Out of then 45 states (including the District of Columbia) with youth sports TBI laws, 14 had references to the NFL in these legislative history sources. ${ }^{204}$ These references were assigned strengths of high, medium, or low, describing the evidence of influence that the NFL had on the legislative process.

High evidence of influence in the legislative process is shown by one or more documents with a direct connection between the state's legislative efforts and the NFL's official lobbying, express support, recommendations, or sponsorship. The term "direct connection" denotes that materials found in the legislative record were either explicitly endorsed or provided to the state legislature by the NFL and reasonably could be considered as evidence of the organization's apparent lobbying for the bill. For example, in Alaska, high evidence of influence included attached documents in the legislative history for Alaska Statutes section 14.30.142, such as a "Youth Concussion Education Pack," distributed to the state legislature and expressly endorsed by the NFL, as well as minutes from the House Education Committee that show the NFL Director Kenneth Edmonds reading a prepared statement into testimony. ${ }^{205}$ Such direct lobbying efforts appeared in ten states. ${ }^{206}$

Medium evidence of influence in the legislative history is shown by references to the NFL in documents that suggest the organization's influence but do not confirm direct involvement in the efforts to pass state laws. The term "suggest" denotes materials found in the legislative record that did explicitly mention the NFL but that were neither clearly printed nor provided to the state legislature by the organization itself. Such materials were assessed as having the potential to influence the legislation without concluding that the NFL played a direct role in lobbying. For example, within the bill analysis and fiscal impact statement for Florida Statutes section 943.0348, a summary history of the Zackery Lystedt law mentions that "Roger Goodell, Commissioner of the National Football League, sent a letter to state governors urging their support of legislation that would better protect young athletes by mandating a more formal and aggressive approach to treatment of concussions. ${ }^{, 207}$ This reference connects the NFL to Florida's legislative efforts but does not clearly indicate either that the letter was sent to Florida's governor or that this legislation was begun on

204 Information about specific references to the NFL in each state's legislative history is on file with the author and available upon request.

205 Student Athlete Traumatic Brain Injuries: Hearing on H.B. 15 Before the H. Educ. Standing Comm., 27th Leg., 1st Sess. 11 (Alaska 2011) (statement of Kenneth Edmonds, Diector, Nat'l Football League).

206 These states are Alaska, California, Delaware, Hawaii, Kansas, Nebraska, Nevada, North Dakota, and Texas, as well as the District of Columbia.

207 Prof'l Staff of the Budget Comm., Bill Analysis and Fiscal Impact Statement, S.B. 256 (Fla. 2012) 
account of such letter. Similar evidence appeared in three states. ${ }^{208}$

Low evidence of influence in the legislative history is shown by a passing and indirect mention of the NFL's connection to the law in the documents available with the bill online. For example, Maryland's legislative record has one mention of the NFL embedded in a summary of background for the bill. Within the fiscal and policy notes is a reference to "the online ImPACT test, which is the same test used by the National Football League."209 Maryland was the only state with such low evidence.

The failure of the vast majority of states to upload supporting documentation and information pertaining to their youth sports TBI laws is worth noting. Whether this lack of policymaking source material is intentional or accidental, it undoubtedly frustrates governmental transparency, as well as this and future attempts at online legislative history research. Thirty-one states have youth sports TBI laws for which the legislative history online reveals no NFL references. ${ }^{210}$ However, lack of evidence is not dispositive of legislative history NFL references for the majority of states, given the absence of any additional information about the legislative process pertaining to those states' youth sports TBI laws.

\section{Appendix 3: NFL Statements Regarding Its Own Legislative Involvement}

The NFL describes its influence in the development of these youth sports TBI laws on its website, which lists legislation by state. ${ }^{211}$ The site was "last updated August 16, 2012," but curiously specifies that "[a]s of October 2012, 40 states (plus the District of Columbia and the city of Chicago) have adopted youth concussion laws." 212 The page goes on to add that "[t]he NFL supports and recognizes the laws as they represent the main principles of the Lystedt Law model legislation...," and lists the three tenets. ${ }^{213}$ The rest of the page lists "Legislative updates on all 50 states." 214 Confusingly, the list includes 44 youth sports TBI laws passed. ${ }^{215}$

From this list, the NFL expresses its support for 34 of the bills, and the states' bill summary bears the marker "Status: Legislation passed with NFL

208 These states are Florida, Michigan, and Washington.

209 See supra note 204.

210 These states are Alabama, Arkansas, Arizona, Colorado, Connecticut, Iowa, Idaho, Illinois, Indiana, Kentucky, Louisiana, Maine, Massachusetts, Minnesota, Missouri, North Carolina, New Hampshire, New Jersey, New Mexico, New York, Ohio, Oklahoma, Oregon, Pennsylvania, Rhode Island, South Dakota, Utah, Virginia, Vermont, Wisconsin, and Wyoming.

211 See Concussion Legislation, supra note 77.

$212 I d$.

$213 \mathrm{Id}$.

$214 \mathrm{Id}$.

$215 \mathrm{Id}$. 
support," with a few minor wording differences among them. ${ }^{216}$

For two states, the NFL specifies that the legislation passed with support from affiliated parties, but not from the organization directly: Illinois, "with support from the Chicago Bears," and Washington, "with Seattle Seahawks' support."217

For eight states, the NFL does not indicate their support in any direct manner. ${ }^{218}$ However, the NFL does include a summary of the legislation, specifying that each includes or contains the "three tenets of model legislation.",219

For Wyoming alone, the NFL does not indicate its support, and furthermore specifies how this youth sports TBI law diverges from the three tenets of the model legislation. ${ }^{220}$ This is consistent with the NFL's uniform promotional approach via the distribution of materials and representatives supporting the model legislation. ${ }^{221}$ The specification of differences between the Wyoming bill and the model legislation could be read to suggest the NFL's disapproval.

The express support of the NFL corresponds extremely well with those states with the strongest news and legislative history NFL references. ${ }^{222}$ Of the 14 states with any legislative history NFL reference at all, all of them except Washington received express NFL support. ${ }^{223}$ Of the 17 states with high-strength news NFL references, all of them except Washington received express NFL support. ${ }^{224}$ This is consistent with the express lobbying efforts of the NFL in relation to these states' bills and the open news coverage of this influence. The Washington exception is easily explained by virtue of its status as the original state from which the model legislation was drawn.

The modified support of the NFL through an affiliated party corresponds with states where there was either medium-(Illinois) or high-(Washington) strength news NFL references. Washington also had medium strength legislative history NFL references.

The absence of expressed NFL support corresponds somewhat with those

216 Id. These states are Alaska, Alabama, Arkansas, California, Colorado, Delaware, Florida, Hawaii, Iowa, Idaho, Indiana, Kansas, Kentucky, Louisiana, Maryland, Maine, Mississippi, Minnesota, Missouri, North Carolina, North Dakota, Nebraska, New Hampshire, New Jersey, Nevada, New York, Ohio, Pennsylvania, South Dakota, Texas, Utah, Vermont, and Wisconsin, as well as the District of Columbia.

217 Id.

218 Id.

219 Id. These states are Connecticut, Massachusetts, New Mexico, Oklahoma, Oregon, Rhode Island, and Virginia.

$220 \mathrm{Id}$.

221 See supra Appendix 2.

222 See supra Appendices 1-2.

223 See supra Appendix 2.

224 Id. 
states having the lowest news and legislative history NFL references. ${ }^{225}$ Six of the eight states receiving no statement of NFL support have medium-strength news references. ${ }^{226}$ The other two states, Oklahoma and Wyoming, had lowstrength news references. ${ }^{227}$ Maryland was the only state with a low-strength legislative history NFL reference and where the NFL expressed support of the state's bill. ${ }^{228}$ Similarly, of the seven states with low news NFL references, only Oklahoma and Wyoming's bills are not expressly supported by the NFL. ${ }^{229}$ Of those 13 states with no news NFL references at all, the NFL page does not recognize Arkansas as having pending or passed relevant legislation at the time of the update, while all the rest received the NFL's express support. ${ }^{230}$

In summary, where the legislative history shows any evidence of the NFL's influence, this is consistent with the NFL's expressed support. Likewise, where the news reports show strong evidence of the NFL's influence, this is consistent with the organization's expressed support. The NFL's lack of expressed support is consistent with those states with low- or medium-strength news references, but not all states with no, low-, or medium-strength news references lacked express NFL support. This is understandable given the limitations of both the press to cover legislative history in depth and the search protocol applied to return all relevant news references.

Ultimately, to whatever extent the NFL has promoted youth sports TBI legislation, it has overwhelmingly succeeded. In states where there are youth sports TBI laws, the NFL has supported a vast majority of them, and it has played an influential role in the legislative history of many. The NFL may have had a similarly strong influence over additional states' legislative efforts, but the records available for research online are either nonexistent or fail to comprehensively disclose this role. 